\title{
Theoretical Analysis and Circuit Verification for Fractional-Order Chaotic Behavior in a New Hyperchaotic System
}

\author{
Ling Liu ${ }^{1,2}$ and Chongxin Liu ${ }^{1,2}$ \\ ${ }^{1}$ State Key Laboratory of Electrical Insulation and Power Equipment, Xian 710049, China \\ ${ }^{2}$ School of Electrical Engineering, Xian Jiaotong University, Xian 710049, China \\ Correspondence should be addressed to Ling Liu; liul@mail.xjtu.edu.cn
}

Received 20 May 2014; Revised 2 August 2014; Accepted 5 August 2014; Published 27 August 2014

Academic Editor: Jun Cheng

Copyright (C) 2014 L. Liu and C. Liu. This is an open access article distributed under the Creative Commons Attribution License, which permits unrestricted use, distribution, and reproduction in any medium, provided the original work is properly cited.

A novel nonlinear four-dimensional hyperchaotic system and its fractional-order form are presented. Some dynamical behaviors of this system are further investigated, including Poincaré mapping, parameter phase portraits, equilibrium points, bifurcations, and calculated Lyapunov exponents. A simple fourth-channel block circuit diagram is designed for generating strange attractors of this dynamical system. Specifically, a novel network module fractance is introduced to achieve fractional-order circuit diagram for hardware implementation of the fractional attractors of this nonlinear hyperchaotic system with order as low as 0.9. Observation results have been observed by using oscilloscope which demonstrate that the fractional-order nonlinear hyperchaotic attractors exist indeed in this new system.

\section{Introduction}

As a very attractive theoretical subject, chaotification which involves some complicated but well-organized dynamical behaviors is a quite challenging topic and has been intensively studied in the last four decades by many pioneer contributors [1-7]. Due to the great effort from these researchers, many classical common chaotic systems and high-dimensional autonomous chaotic systems have been investigated through theoretical analysis and numerical simulation [8-10]. Meanwhile, it is noticed that observation of chaotic dynamical behaviors in the experiments has begun to attract much attention in recent years. In particular, generating chaotic attractors by using hardware implementation has been a subject of interest because of their potential applications in various chaos-based technologies, such as encryption and secure communications. In secure communication, for example, a signal typically from a common chaotic system was suited to mask a message to be transmitted [11, 12].

More recently, with rapid advances in nonlinear circuit theory, many sophisticated electronic circuits have been designed to exhibit complexity behaviors of chaos. To begin with, it is necessary to briefly review some main advances in three-dimensional chaotic attractor and high-dimensional (hyperchaos) attractor generation by using hardware circuit design. In [13], the authors proposed that the hyperchaos can be generated by coupled Colpitts oscillators. In [14], a new hyperchaotic Lorenz system is illustrated not only by computer simulation but also by the realization of an electronic circuit. Furthermore, the design and circuit implementation of some new hyperchaotic and four-dimensional chaotic oscillators have been introduced in detail [15-20]. In particular, many investigations are devoted to research the dynamical properties of fractional-order chaotic systems very recently.

Although fractional derivatives as a pure mathematical topic have a long history, the applications of this topic to physics and engineering are just a recent focus of interest. According to the classical point of Poincare-Bendixson theorem, it is well known that chaos phenomenon cannot occur in dimensions less than three of autonomous equations. Therefore, by using fractional derivatives of orders 
$0<\alpha_{i}<1$, it is possible to obtain chaotic attractors with an effective dimension $\sum<3$. Historically, Hartley et al. first discovered chaotic motion of the famous Chua circuit system of a fractional-order form with order as low as 2.7 [21]. I. Grigorenko and E. Grigorenko introduced a generalization of the Lorenz dynamical system using fractional derivatives [22]. In [23], some chaotic behaviors in a fractional-order modified Duffing system have been studied by numerical analysis. Hyperchaos in fractional-order Rossler system is being investigated in detail in [24]. In [25], the chaotic behavior of the fractional unified system has been studied with lowest order from 2.97 to 2.07. However, it is rather rare to observe a common chaos or high-dimensional chaos in their fractional-order form. As a result, there are very few reports on generating common chaos especially highdimensional chaotic attractors in the literature.

Moreover, generating a nonlinear chaotic attractor from fractional-order dynamical system by using physical circuit is quite difficult. Up to now, there are two modules of fractional circuit for designing the chaotic oscillation circuit. The first example of an electrical circuit related to fractional calculus is called a tree fractance circuit element, where an infinite self-similar circuit consists of $N$ resistor $R$ and capacitors $C$ [26]. The second example is called chain fractance circuit which also consists of $N$ resistor-capacitor pairs connected in an infinite chain [27]. In [28], the authors presented a novel three-channel circuit for creating common chaotic attractors with chain fractance. In [29], a remarkable fractional-order circuit is designed for physically verifying a new hyperchaotic system. In particular, the modified four-dimensional Chua's system with its fractional-order attractors has been proofed by circuit realization [30]. As far as we know, previous works on circuit generating fractional attractors only focused on using tree and chain fractance module [31]. Therefore, it is very interesting to ask whether there exists some different topological fractance for circuit design which can generate fractional chaos and hyperchaos $[32,33]$.

In this letter, a new smooth four-dimensional nonlinear autonomous hyperchaotic system has been introduced by adding a controller into the classical Chua's chaotic system with an appropriate cubic nonlinearity. Some basic dynamical properties of this nonlinear dynamical system have been investigated in detail. Furthermore, a fourth-channel circuit diagram is constructed for generating a chaotic attractor. In particular, utilizing the fractional derivatives, a novel network module fractance has been introduced by designing a fractional-order circuit for physically realizing the strange attractors of the fractional-order form of this new hyperchaotic system.

\section{Dynamical Behaviors of the Nonlinear Hyperchaotic System}

For comparison convenience, the mathematical model of the generalized Chua's circuit system should be reviewed by the following expression:

$$
\frac{d x_{1}}{d t}=\beta_{1}\left(x_{2}-F\left(x_{1}\right)\right)
$$

$$
\begin{aligned}
& \frac{d x_{2}}{d t}=\beta_{2} x_{1}-x_{2}+x_{3}, \\
& \frac{d x_{3}}{d t}=-\beta_{3} x_{2}+\beta_{4} x_{3},
\end{aligned}
$$

where $x=\left[x_{1}, x_{2}, x_{3}\right]^{T} \in R^{3}$ is the state vector, $\beta_{1}, \beta_{2}, \beta_{3}$, and $\beta_{4}$ are positive constant parameters, and $F(\cdot)$ is the smooth function that contains cubic nonlinearity.

Based on the above classical system (1), a fourdimensional hyperchaotic should be considered as follows:

$$
\begin{aligned}
& \frac{d x_{1}}{d t}=\beta_{1}\left(x_{2}+0.2\left(x_{1}-2 x_{1}^{3}\right)\right), \\
& \frac{d x_{2}}{d t}=\beta_{2} x_{1}-x_{2}+x_{3}+x_{4}, \\
& \frac{d x_{3}}{d t}=-\beta_{3} x_{2}+x_{4} \\
& \frac{d x_{4}}{d t}=-\beta_{4} x_{1}
\end{aligned}
$$

where $x=\left[x_{1}, x_{2}, x_{3}, x_{4}\right]^{T} \in R^{4}$ is the state vector; $d x_{4} / d t=$ $-\beta_{4} x_{1}$ is the state feedback controller; $\beta_{1}, \beta_{2}, \beta_{3}$, and $\beta_{4}$ are positive constant parameters; and smooth nonlinear function $F(\cdot)$ can be denoted as $F(\cdot)=0.2\left(x_{1}-2 x_{1}^{3}\right)$.

2.1. Dynamical Properties Analysis. In order to reveal dynamical properties of this nonlinear hyperchaotic system, the equilibria of system (2) should be considered at first:

$$
\begin{gathered}
\beta_{1}\left(x_{2}+0.2\left(x_{1}-2 x_{1}^{3}\right)\right)=0 \\
\beta_{2} x_{1}-x_{2}+x_{3}+x_{4}=0 \\
-\beta_{3} x_{2}+x_{4}=0 \\
-\beta_{4} x_{1}=0
\end{gathered}
$$

The only one equilibrium of system (2) has been obtained, which is expressed as $E_{0}(0,0,0,0)$. It can be found out that the number of equilibria points of system (2) is significantly less than the classical three- or four-dimensional nonlinear system. For system (2), the corresponding Jacobian matrices can be written in the form

$$
J_{\left(x_{1}, x_{2}, x_{3}, x_{4}\right)}=\left(\begin{array}{cccc}
0.2 \beta_{1}\left(1-6 x_{1}^{2}\right) & \beta_{1} & 0 & 0 \\
\beta_{2} & -1 & 1 & 1 \\
0 & -\beta_{3} & 0 & 1 \\
-\beta_{4} & 0 & 0 & 0
\end{array}\right)
$$

when we choose $\beta_{1}=8.25, \beta_{2}=0.8, \beta_{3}=12.25$, and $\beta_{4}=0.2$, the Jacobian system, evaluated at $E_{0}(0,0,0,0)$, is given by

$$
J_{0}=\left(\begin{array}{cccc}
1.65 & 8.25 & 0 & 0 \\
0.8 & -1 & 1 & 1 \\
0 & -12.25 & 0 & 1 \\
-0.2 & 0 & 0 & 0
\end{array}\right)
$$


The way to solve for eigenvalues as roots of the characteristic equation is $\left|\lambda I-J_{0}\right|=0$. Eigenvalues at $E_{0}(0,0,0,0)$ are obtained as $\lambda_{1}=2.5262, \lambda_{2}=0.0899, \lambda_{3}=-0.983+$ i2.51, and $\lambda_{4}=-0.983-i 2.51$. It is easy to see that $\lambda_{1}$ and $\lambda_{2}$ are two positive real roots and $\lambda_{3}$ and $\lambda_{4}$ are a pair of complex conjugate eigenvalues with negative real parts. In this case, the equilibrium of system (2) is an unstable saddlefocus point. According to nonlinear dynamical theory, the Jacobian should have at least one unstable eigenvalue that the nonlinear system can display chaotic behaviors.

Remark 1. Assume that $\Omega$ denotes a region in $R^{4}$ with a smooth boundary $\partial \Omega$. Let $\Omega(t)$ be the region formed by flowing along for time $t$ and then let $\Omega(t)=\left\{\Phi\left(t ; x_{0}\right)\right.$ : $\left.x_{0} \in \Omega\right\}$. For system (2), the phase space is determined by the divergence of the flow $\dot{V}(t)=\int_{\Omega(t)} \nabla F d V$ which gives $\dot{V}<0$.

The general condition of dissipative can be gained as

$$
\nabla V=\frac{\partial \dot{x}_{1}}{\partial x_{1}}+\frac{\partial \dot{x}_{2}}{\partial x_{2}}+\frac{\partial \dot{x}_{3}}{\partial x_{3}}+\frac{\partial \dot{x}_{4}}{\partial x_{4}}=-\delta=0.2 a-1.2 a x^{2}-1
$$

with $x>\sqrt{13 / 198}$, since $V(t)=V(0) e^{\left(0.2 a-1.2 a x^{2}-1\right) t}$ is the volume element of $\Omega(t)$. Apparently, the volume of $V(t)$ will be decreased exponentially fast. Therefore, this means that each volume containing the system trajectory shrinks to zero as $t \rightarrow \infty$ at an exponential rate $V(0) e^{-\delta t}$. Ultimately, all trajectories of system (2) are confined to a subset of zero volume and the asymptotic motion settles onto a strange attractor.

According to the nonlinear dynamical theory, a quantitative measure approach of the sensitive dependence on the initial conditions is calculating the Lyapunov exponent. It is the average rate of divergence (or convergence) of two neighboring trajectories. Moreover, the corresponding Lyapunov exponents of the nonlinear dynamical system (2) with parameters $\beta_{1}=10, \beta_{2}=1, \beta_{3}=12.5$, and $\beta_{4}=5$ are obtained as follows:

$$
\begin{array}{cl}
\lambda_{L_{1}}=0.2458, & \lambda_{L_{2}}=0.0175, \\
\lambda_{L_{3}}=-0.0398, & \lambda_{L_{4}}=-3.2881 .
\end{array}
$$

It is easy to see that there are two positive exponents $\lambda_{L_{1}}$ and $\lambda_{L_{2}}$. According to Wolf method if one nonlinear system has more than one positive Lyapunov exponent, then that indicates that the system may be hyperchaotic with complex dynamical behaviors.

Furthermore, the Lyapunov dimension of system (2) can be calculated as follows:

$$
\begin{aligned}
D_{L} & =j+\frac{1}{\left|\lambda_{L_{j+1}}\right|} \sum_{i=1}^{j} \lambda_{L_{j}}=2+\frac{\left(\lambda_{L_{1}}+\lambda_{L_{2}}\right)}{\left|\lambda_{L_{3}}+\lambda_{L_{4}}\right|} \\
& \approx 2+\frac{0.2458+0.0175}{-0.0398-3.2881} \approx 2.08 .
\end{aligned}
$$

2.2. Nonlinear Circuit Verification for Hyperchaotic System (2). In this subsection, a nonlinear fourth-order oscillation circuit diagram has been designed for physically verifying the four-dimensional hyperchaotic system (2). In order to obtain the circuit diagram we should transform the dimensionless equations of system (2)into the following suitable $R-C$ 


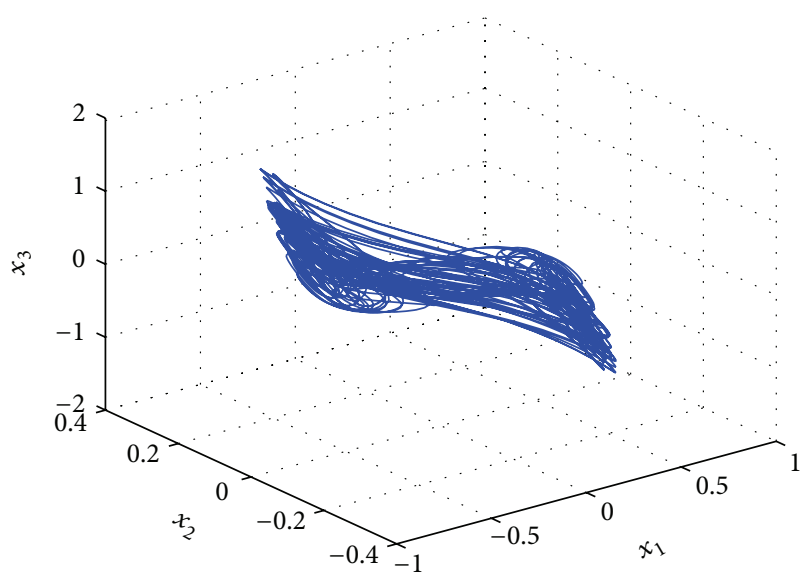

(a)

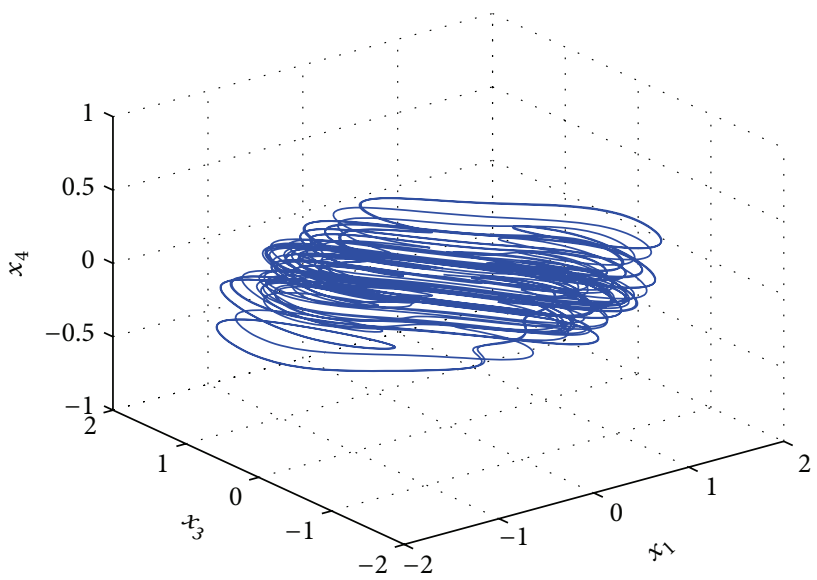

(c)

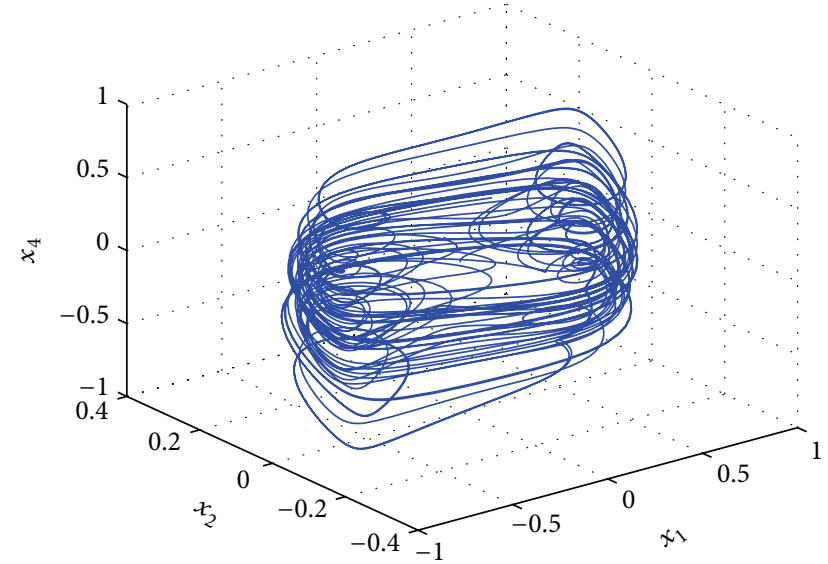

(b)

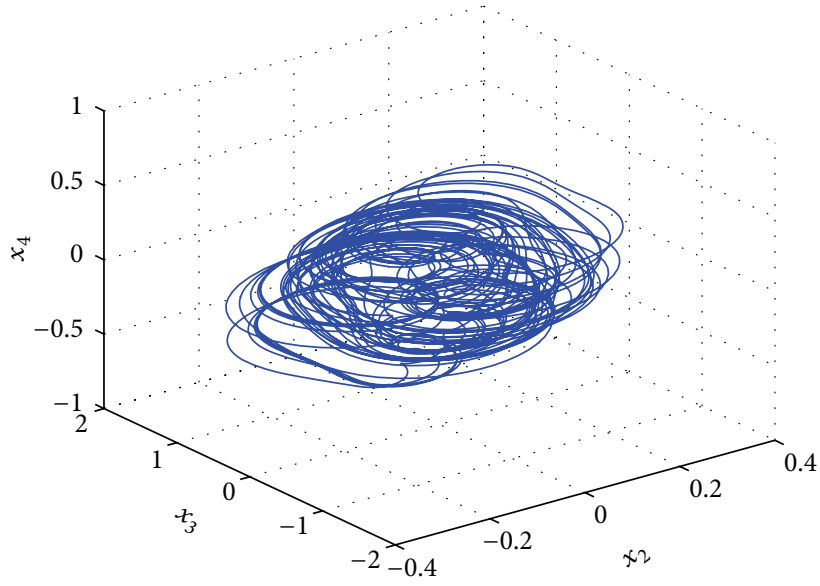

(d)

FIgURE 2: Phase portraits of the hyperchaotic attractors in three quadrants.

equations form:

$$
\begin{aligned}
\frac{d u_{C_{1}}}{d t}= & \frac{R_{2}}{R_{3} R_{7} C_{1}} u_{C_{2}}+\frac{R_{2}}{R_{3} C_{1}}\left(\frac{1}{R_{6}} u_{C_{1}}-\frac{R_{5}}{100 R_{1} R_{4}} u_{C_{1}}^{3}\right), \\
\frac{d u_{C_{2}}}{d t}= & \frac{R_{9}}{R_{8} R_{10} C_{2}} u_{C_{1}}-\frac{R_{9} R_{12}}{R_{10} R_{11} R_{13} C_{2}} u_{C_{2}}+\frac{R_{9}}{R_{10} R_{14} C_{2}} u_{C_{3}} \\
& +\frac{R_{9}}{R_{10} R_{18}} u_{C_{4}}, \\
\frac{d u_{C_{3}}}{d t}= & -\frac{R_{12} R_{16}}{R_{11} R_{15} R_{17} C_{3}} u_{C_{2}}+\frac{R_{16}}{R_{17} R_{22} C_{3}} u_{C_{4}}, \\
\frac{d u_{C_{4}}}{d t}= & -\frac{R_{12} R_{20}}{R_{11} R_{19} R_{21} C_{4}} u_{C_{1}},
\end{aligned}
$$

where state variables $x_{1}, x_{2}, x_{3}$, and $x_{4}$ can be generated from $v_{C_{1}}, v_{C_{2}}, v_{C_{3}}$, and $v_{C_{4}}$, which are the voltage outputs over the capacitors. The circuit diagram includes ten operational amplifiers LM741 chip (the power supply is set to be 15 \pm ), two analog multiplier AD633 chips, four capacitors $\left(C_{1} \sim C_{4}\right)$, and some linear resistors $\left(R_{1} \sim R_{22}\right)$. Figure 6 shows the circuit diagram. All resistors are exactly adjustable resistors with high precision. Resistance is as follows: $R_{1}=5 \mathrm{k} \Omega ; R_{2}$, $R_{9}, R_{20}=1 \mathrm{k} \Omega ; R_{3}, R_{4}, R_{5}, R_{7}, R_{11}, R_{12}, R_{19}=10 \mathrm{k} \Omega$; $R_{6}=50 \mathrm{k} \Omega ; R_{8}, R_{13}, R_{14}, R_{18}, R_{22}=250 \mathrm{k} \Omega ; R_{10}=4 \mathrm{k} \Omega$; $R_{15}, R_{21}=20 \mathrm{k} \Omega ; R_{16}=25 \mathrm{k} \Omega$. Capacitance is as follows: $C_{1}$, $C_{2}, C_{3}, C_{4}=1 \mu \mathrm{F}$.

Figures 7(a) to 7(f) show the experimental observation of these hyperchaotic strange attractors on $x_{1}-x_{2}, x_{1}-x_{3}, x_{1}-$ $x_{4}, x_{1}-x_{4}, x_{2}-x_{3}, x_{2}-x_{4}$, and $x_{3}-x_{4}$ phase plane, respectively. Comparing the simulation results and experimental results, it can be declared that a very good qualitative agreement has been confirmed.

\section{Fractional-Order Form of Hyperchaotic System (2)}

3.1. Fractional Numerical Algorithm. In this section, the dynamical equations of noninteger (fractional-order) form of system (2) have been constructed at first. 


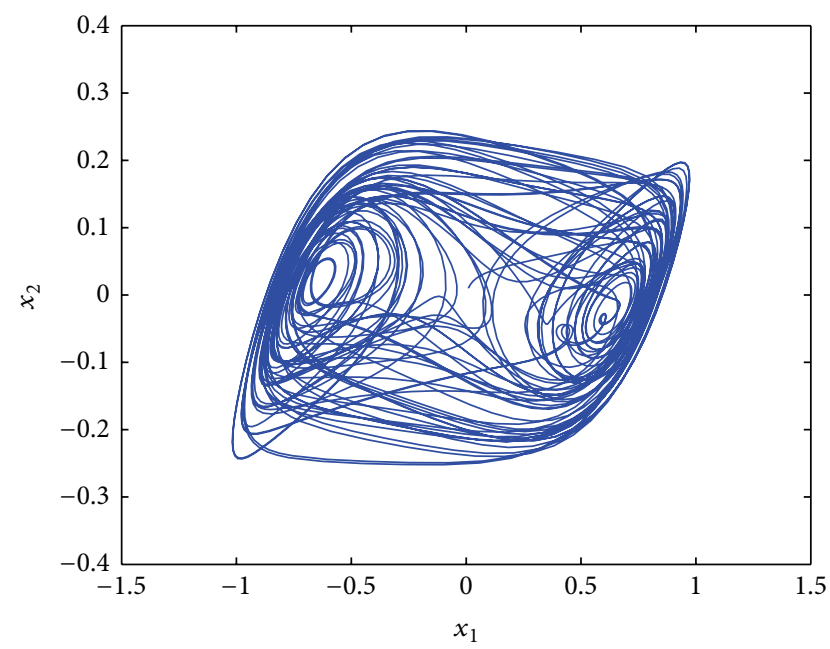

(a)

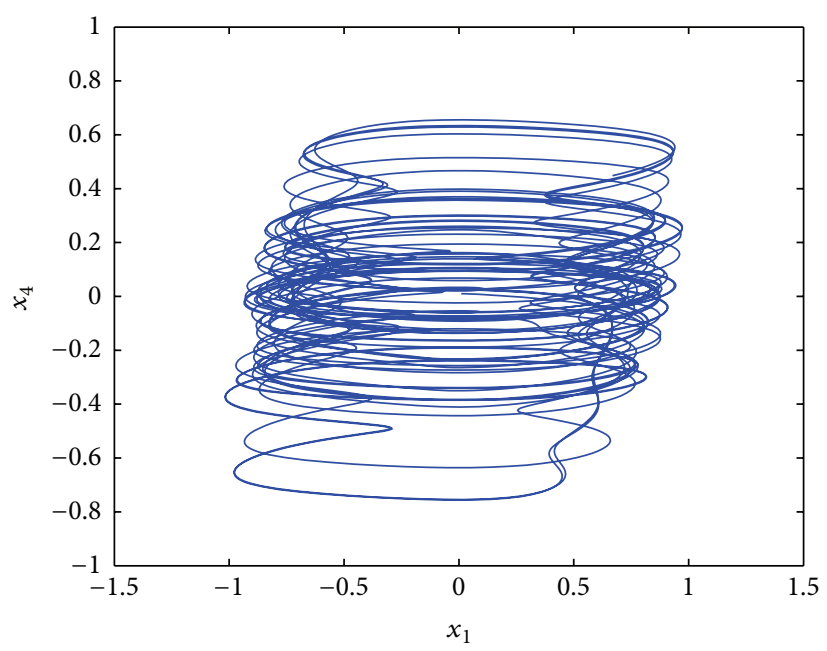

(c)

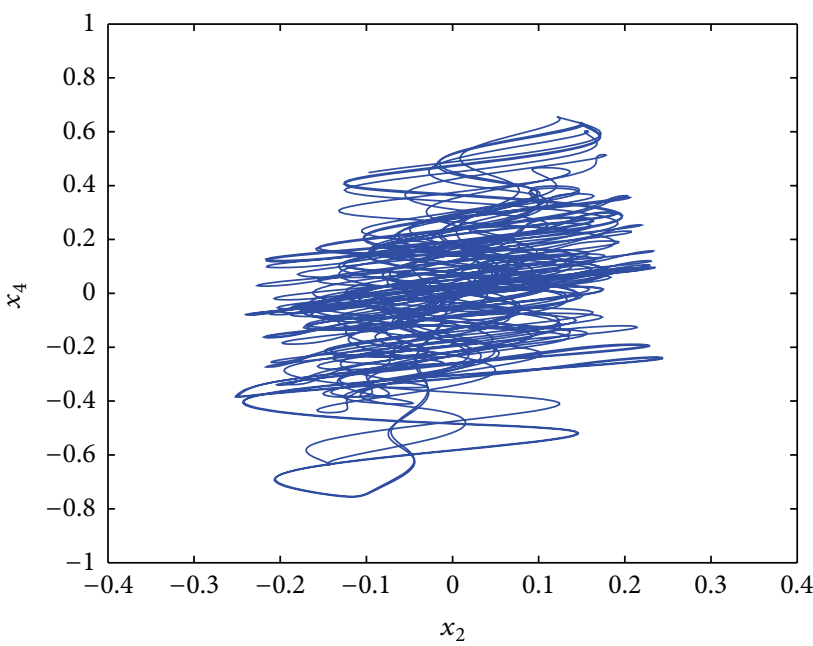

(e)

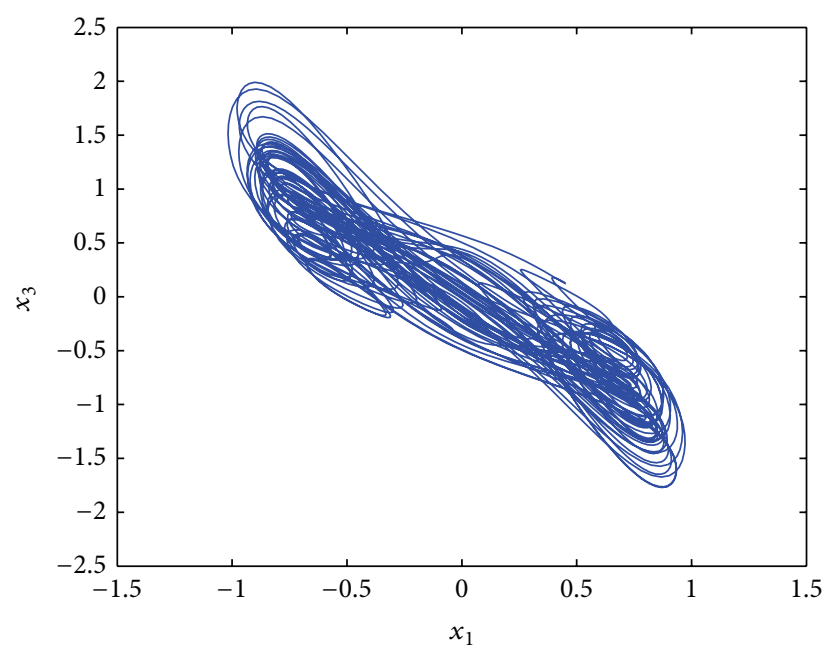

(b)

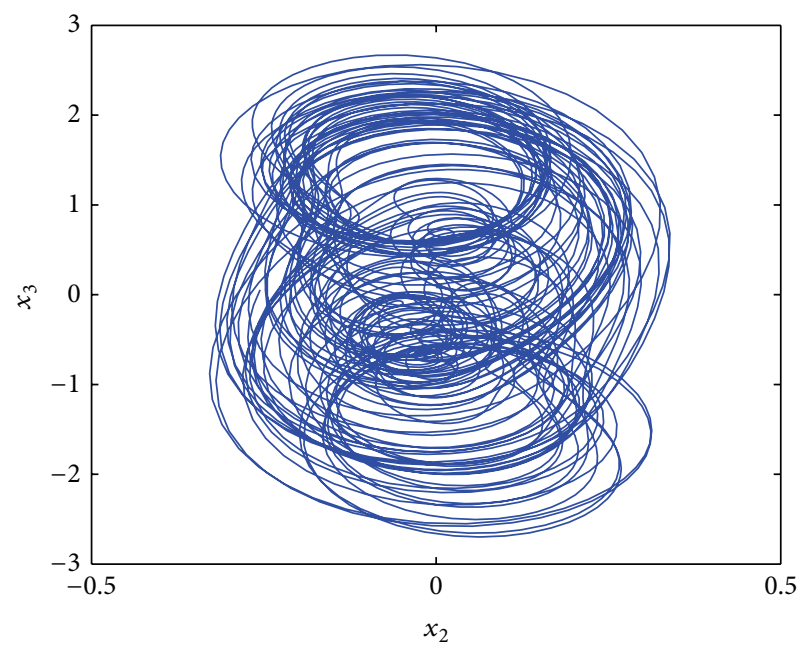

(d)

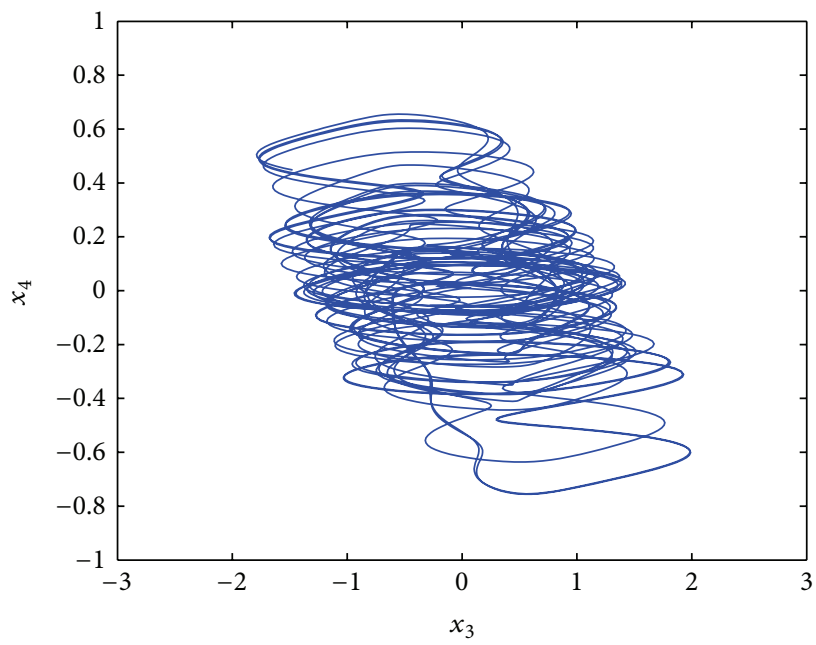

(f)

FIGURE 3: Phase portraits of the hyperchaotic attractors on $x_{1}-x_{2}, x_{1}-x_{3}, x_{1}-x_{4}, x_{2}-x_{3}, x_{2}-x_{4}$, and $x_{3}-x_{4}$ plane projection. 


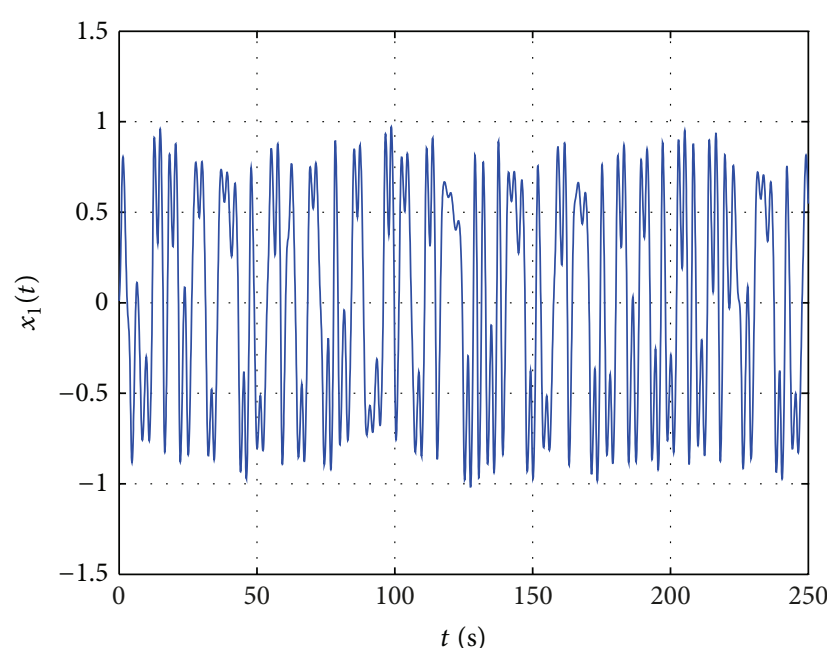

(a)

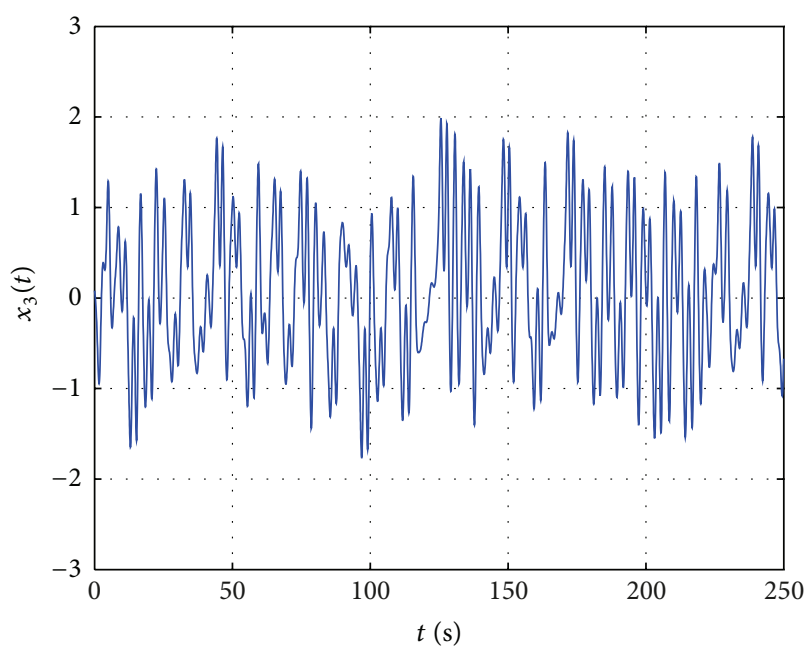

(c)

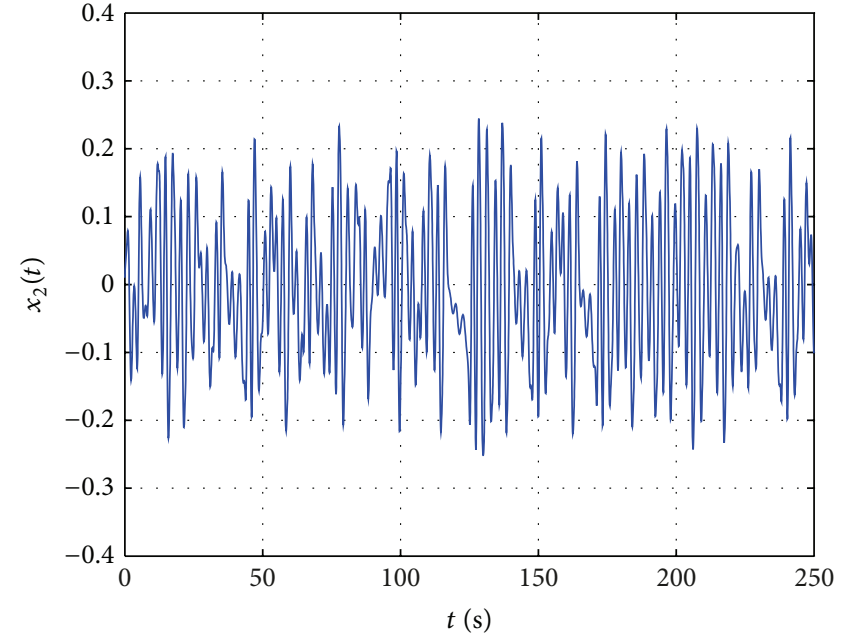

(b)

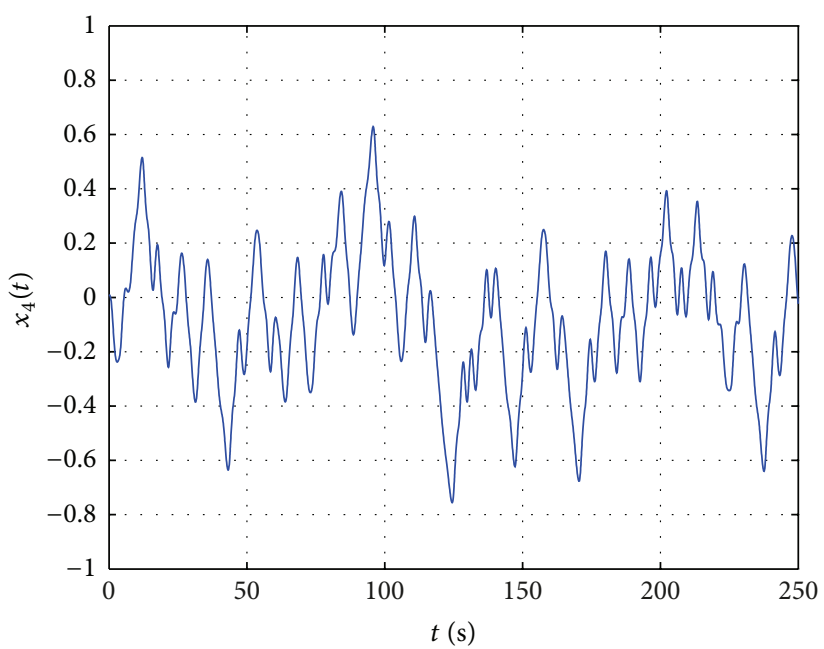

(d)

FIGURE 4: The time waveform response of the states $x_{1}, x_{2}, x_{3}$, and $x_{4}$ of the system (2).

Although the theory of fractional derivatives developed as a pure mathematical topic that has three centuries, its applications did not attract much attention until the recent decades. There are several definitions of fractional derivatives that are well known. Three commonly used definitions for the general fractional differintegral nonlinear systems are the Riemann-Liouville (RL), Grünwald-Letnikov (GL), and Caputo's definition.

The uniform formula of a fractional derivative with $\alpha \epsilon$ $(0,1)$ can be defined as follows:

$$
{ }_{\alpha} D_{t}^{-\alpha}=\frac{1}{\Gamma(\alpha)} \int_{a}^{t} \frac{f(\tau)}{(t-\tau)^{1-\alpha}} d \tau
$$

where $f(\cdot)$ is an arbitrary integrable smooth function and ${ }_{\alpha} D_{t}^{-\alpha}$ is the fractional derivative of order $\alpha$ on $[a, t]$. Consider

$$
\Gamma(z)=\int_{0}^{\infty} e^{-t} t^{z-1} d t
$$

$$
\begin{aligned}
& =\int_{0}^{1} e^{-t} t^{z-1} d t+\int_{1}^{\infty} e^{-t} t^{z-1} d t \\
& =\sum_{k=0}^{\infty} \frac{(-1)^{n}}{n !(n+z)}+\int_{1}^{\infty} e^{(z-1) \log (t)-t} d t .
\end{aligned}
$$

There are several famous definitions of fractional derivatives. Three commonly used definitions for the general fractional differintegral nonlinear systems are the GrünwaldLetnikov (GL), Riemann-Liouville (RL), and Caputo's definition.

The Grünwald-Letnikov (GL) definition can be written as

$$
\begin{aligned}
{ }_{a} D_{t}^{\alpha} f(t) & =\frac{d^{\alpha} f(t)}{d t^{\alpha}}=\lim _{h \rightarrow 0} h^{-\alpha} \sum_{j=0}^{[(t-\alpha) / h]}(-1)^{j}\left(\begin{array}{c}
\alpha \\
j
\end{array}\right) f(t-j h) \\
& =\lim _{h \rightarrow 0} f_{h}^{\alpha}(t),
\end{aligned}
$$




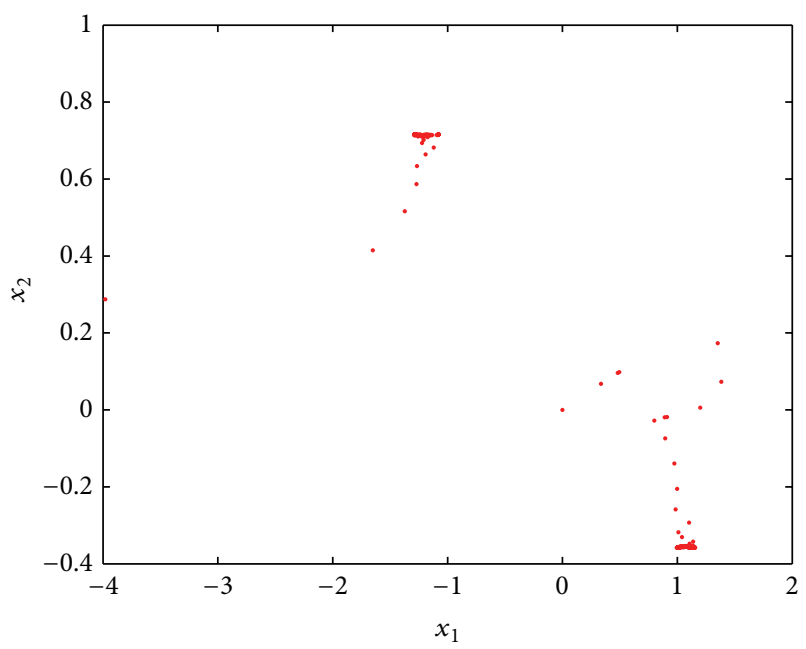

(a)

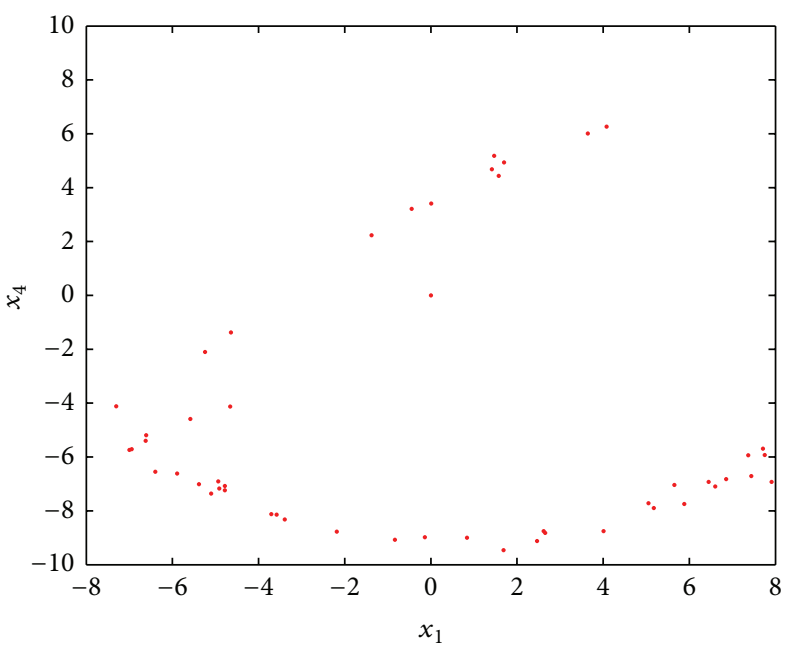

(c)

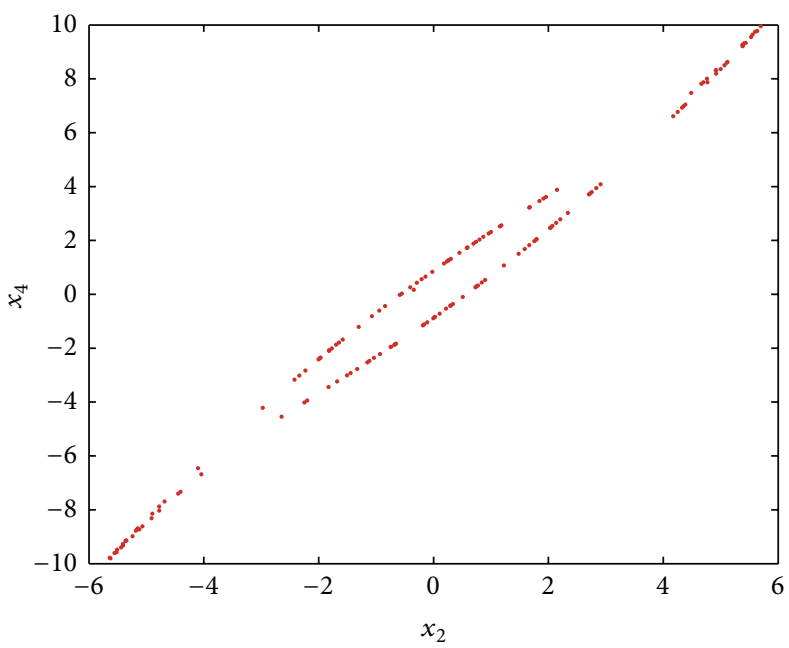

(e)

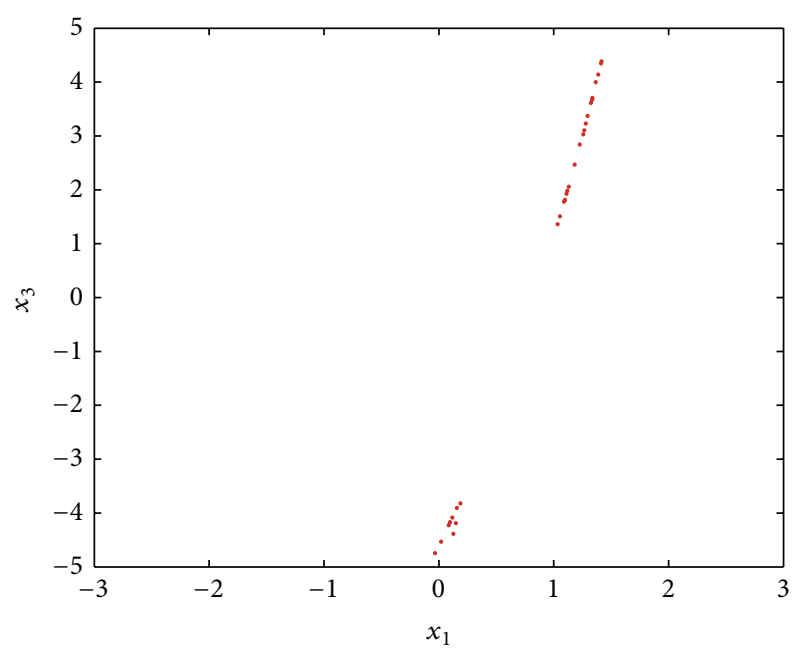

(b)

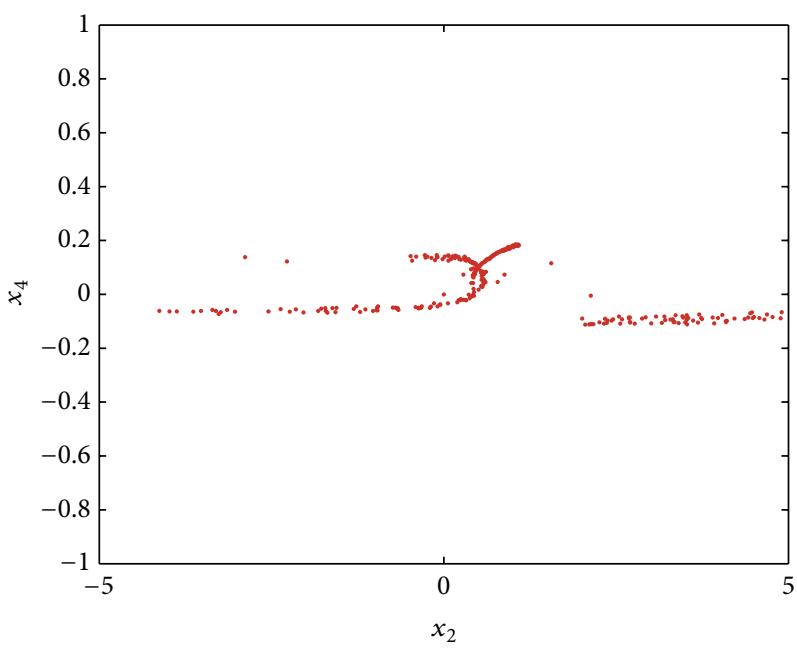

(d)

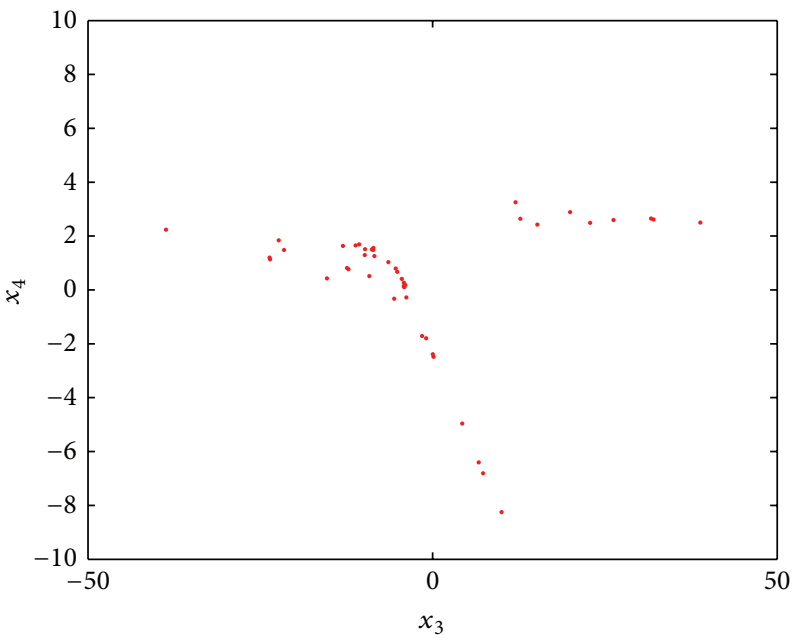

(f)

Figure 5: Poincaré map of the hyperchaotic in different phase plane. 


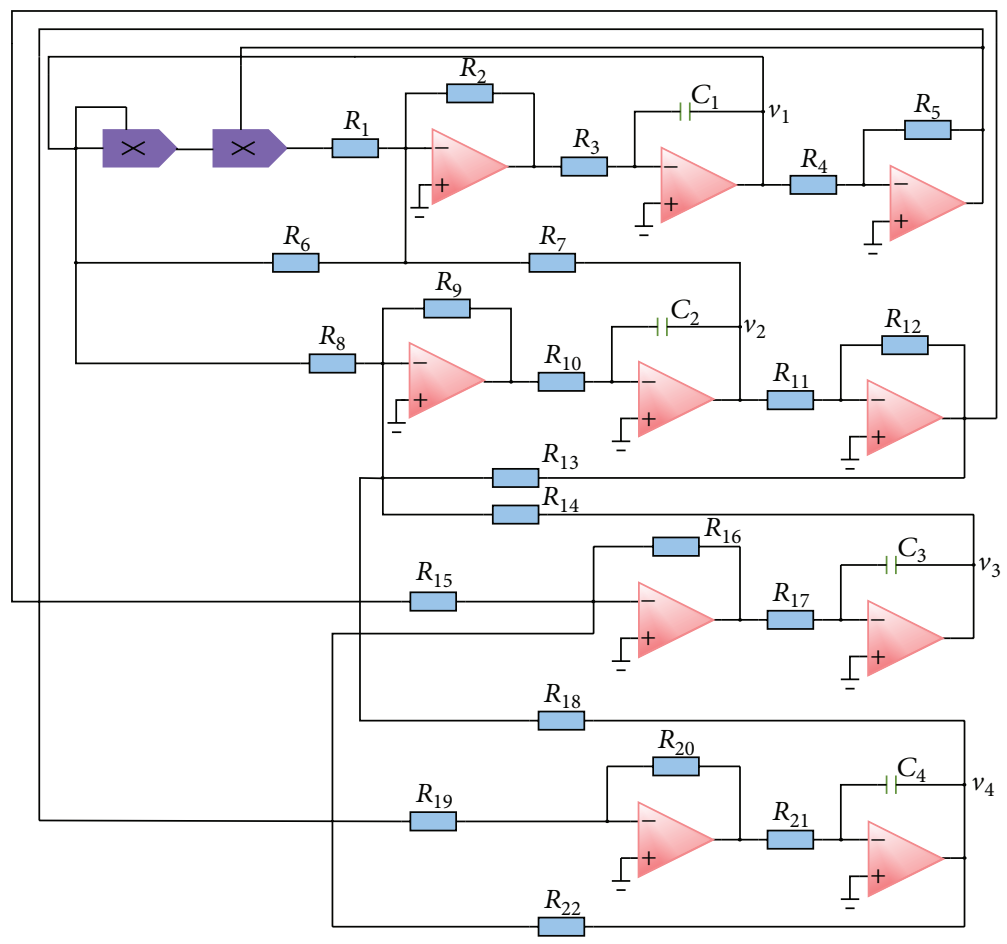

FIGURE 6: Circuit diagram for implementing the hyperchaotic attractors.

where $f_{h}^{(\alpha)}(t)=\lim _{h \rightarrow 0} h^{-\alpha} \sum_{j=0}^{[(t-\alpha) / h]}(-1)^{j}\left(\begin{array}{l}\alpha \\ j\end{array}\right) f(t-j h)$ and $(\cdot)$ means the integer part.

The elegant Riemann-Liouville (RL) fractional derivative is expressed as

$$
{ }_{a} D_{t}^{\alpha} f(t)=\frac{d^{\alpha} f(t)}{d t^{\alpha}}=\frac{1}{\Gamma(n-\alpha)} \frac{d^{n}}{d t^{n}} \int_{a}^{t} \frac{f(\tau)}{(t-\tau)^{\alpha-n+1}} d \tau,
$$

where $n$ is an integer larger than $\alpha$, that is, $n-1<\alpha<n$, and $\Gamma(\cdot)$ is the gamma function.

Caputo's definition can be written as

$$
{ }_{a} D_{t}^{\alpha} f(t)=\frac{d^{\alpha} f(t)}{d t^{\alpha}}=\frac{1}{\Gamma(m-\alpha)} \int_{a}^{t} \frac{f(\tau)}{(t-\tau)^{\alpha-m+1}} d \tau,
$$

where $m$ is an integer larger than $\alpha$, that is, $m-1<\alpha<m$, and $\Gamma(\cdot)$ also is the gamma function.

From (13), the fractional-order form of hyperchaotic system (2) can be obtained as

$$
\begin{aligned}
& \frac{d^{\alpha} x_{1}}{d t^{\alpha}}=\beta_{1}\left(x_{2}+0.2\left(x_{1}-2 x_{1}^{3}\right)\right), \\
& \frac{d^{\alpha} x_{2}}{d t^{\alpha}}=\beta_{2} x_{1}-x_{2}+x_{3}+x_{4}, \\
& \frac{d^{\alpha} x_{3}}{d t^{\alpha}}=-\beta_{3} x_{2}+x_{4}, \\
& \frac{d^{\alpha} x_{4}}{d t^{\alpha}}=-\beta_{4} x_{1},
\end{aligned}
$$

where $\alpha$ is the fractional-order satisfying $0<\alpha<1$.
As is well known, the fractional differintegral does not allow the direct implementation of the fractional operators. Therefore, two approximation methods have been developed for numerical solution and physic realized fractional differential system in the literature. One is the time-domain method which is a generalization of the Adams-Bashforth-Moulton (ABM) predictor-corrector algorithm. The other is by utilizing frequency-domain techniques based on Bode diagrams. One can obtain a linear approximation of a fractional-order integrator with any desired accuracy over any frequency band. In this work, we use the predictor-corrector scheme for numerical simulation and the frequency-based method for fractional circuit implementation.

The (ABM) predictor-corrector scheme for (12) is derived here for numerical simulation analysis. Consider the initial value form of general fractional dynamical system as follows:

$$
\begin{gathered}
D_{*}^{\alpha_{i}} x_{i}(t)=\frac{d^{\alpha_{i}} x_{i}(t)}{d t^{\alpha_{i}}}=f\left(t, x_{i}(t)\right), \\
x^{k}(0)=x_{0}^{(k)}, \quad k=0,1, \ldots,[\alpha]-1 .
\end{gathered}
$$

Note that the differential equation (13) is equivalent to the Volterra integral equation:

$$
x(t)=\sum_{k=0}^{[\alpha]-1} \frac{t^{k}}{k !} x_{0}^{(k)}+\frac{1}{\Gamma(\alpha)} \int_{0}^{t}(t-\tau)^{\alpha-1} f(\tau, x(\tau)) d \tau
$$




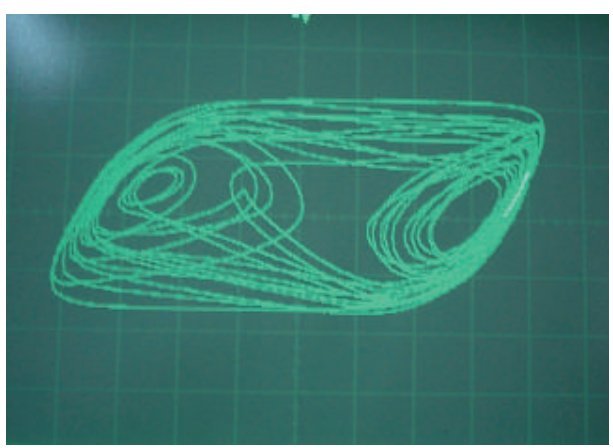

(a) $x_{1}-x_{2}$

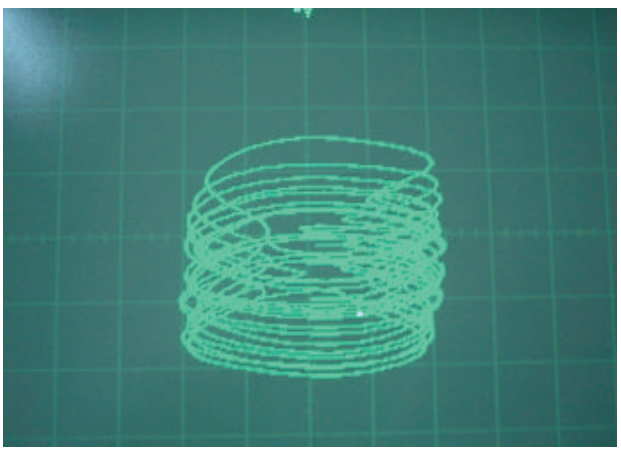

(c) $x_{1}-x_{4}$

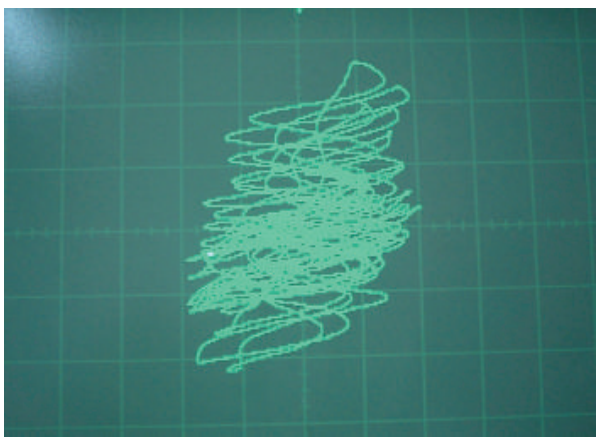

(e) $x_{2}-x_{4}$

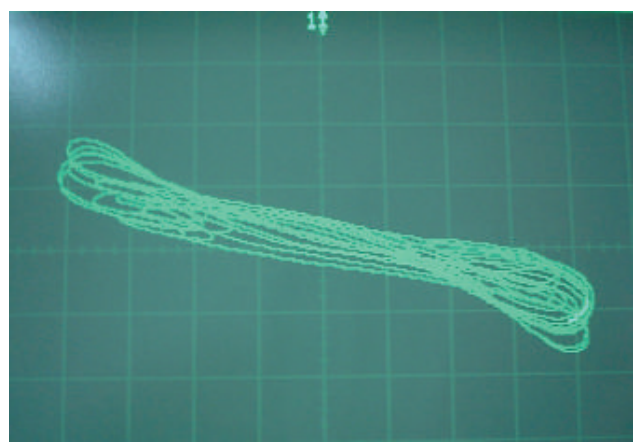

(b) $x_{1}-x_{3}$

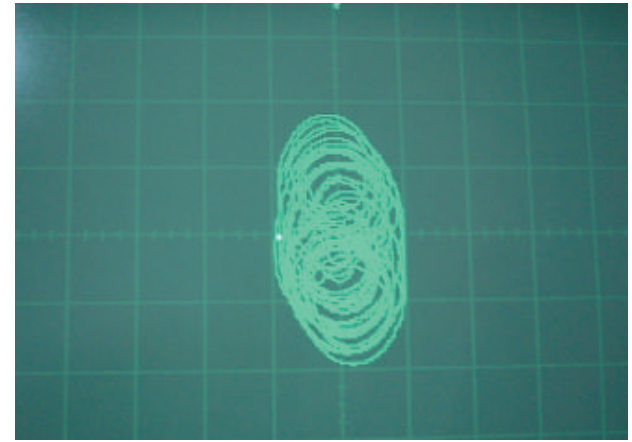

(d) $x_{2}-x_{3}$

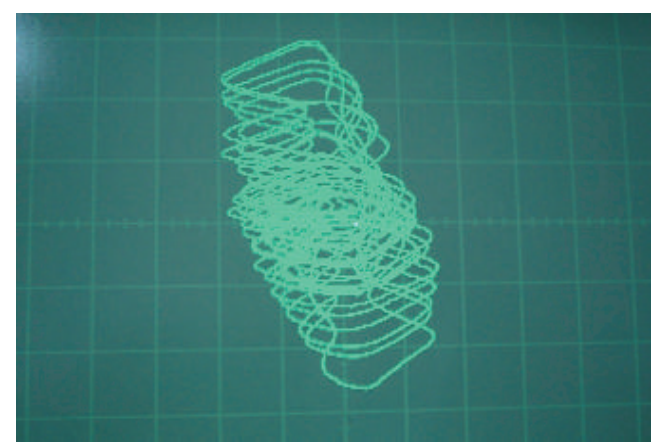

(f) $x_{3}-x_{4}$

FIGURE 7: Experimental observations of the hyperchaotic attractors.

Set $h=T / N$ and $t_{j}=j h,\left(j=0,1, \ldots, N \in Z^{+}\right)$; the corresponding discretization equation for (21) is defined by

$$
\begin{aligned}
x_{h}\left(t_{n+1}\right)= & \sum_{k=0}^{[\alpha]-1} x_{0}^{(k)} \frac{t_{n+1}^{k}}{k !}+\frac{h^{\alpha}}{\Gamma(\alpha+2)} f\left(t_{n+1}, x_{h}^{p}\left(t_{n+1}\right)\right) \\
& +\frac{h^{\alpha}}{\Gamma(\alpha+2)} \sum_{j=0}^{n} a_{j, n+1} f\left(t_{j}, x_{h}\left(t_{j}\right)\right),
\end{aligned}
$$

where

$$
a_{(j, n+1)} \begin{cases}n^{\alpha+1}-(n-\alpha)(n+1)^{\alpha}, & j=0, \\ (n-j+2)^{\alpha+1}+(n-j)^{\alpha+1}-2(n-j+1)^{\alpha+1}, & 1 \leq j \leq n, \\ 1, & j=n+1 .\end{cases}
$$

The predictor $x_{\left(t_{n+1}\right)}^{p}$ is given by

$$
x_{h}^{p}\left(t_{n+1}\right)=\sum_{k=0}^{n+1} \frac{t_{n+1}^{k}}{k !} x_{0}^{k}+\frac{1}{\gamma(\alpha)} \sum_{j=0}^{n} f\left(t_{j}, x_{h}\left(t_{j}\right)\right),
$$

where

$$
b_{(j, n+1)}=\frac{h^{\alpha}}{\alpha}\left((n+1-j)^{\alpha}-(n-j)^{\alpha}\right), \quad 0 \leq j \leq n .
$$

Comparing (15) and (17), estimation error of the approximation is

$$
\begin{gathered}
e^{*}=\max _{j=0,1, \ldots, N}\left|x\left(t_{j}\right)-x_{h}\left(t_{j}\right)\right|=O\left(h^{p}\right), \\
p=\min (2,1+\alpha) .
\end{gathered}
$$




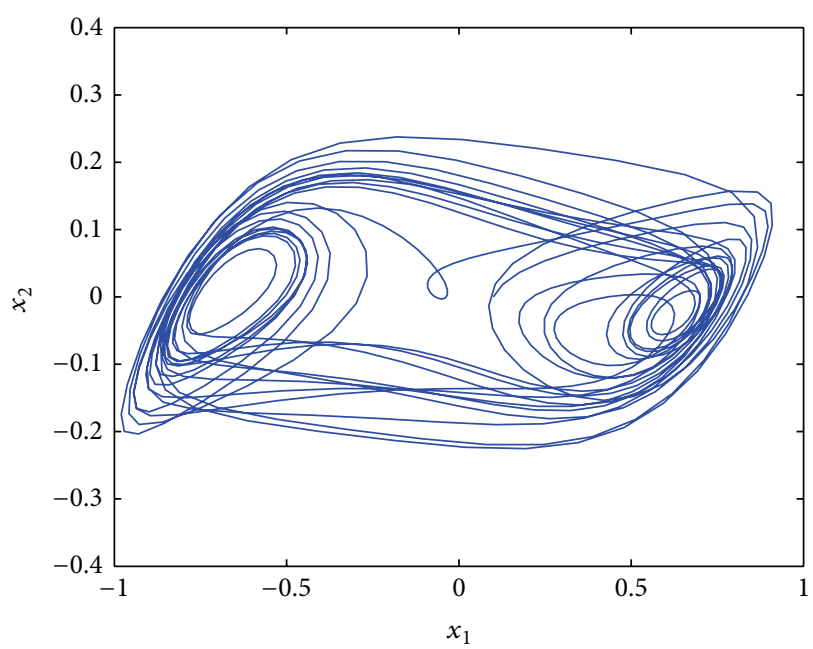

(a)

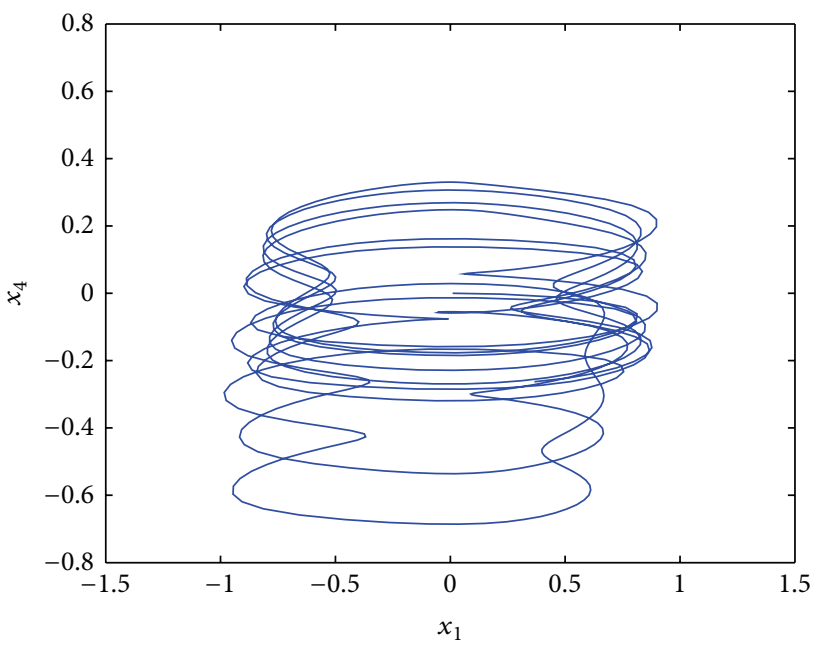

(c)

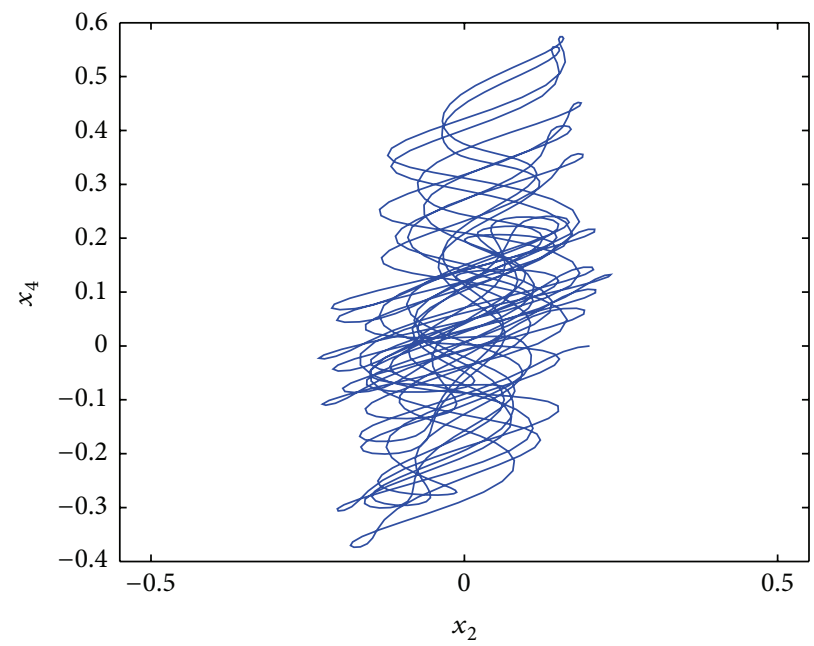

(e)

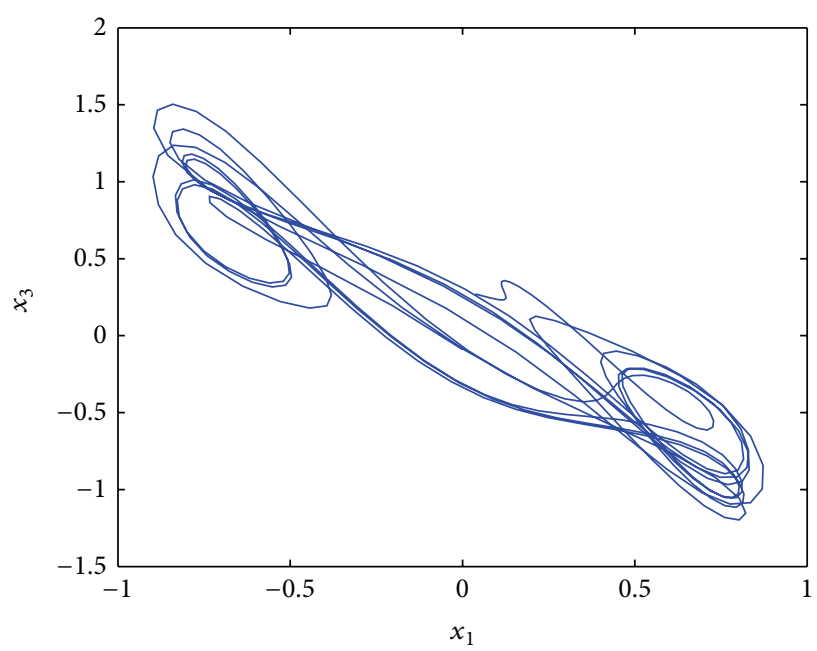

(b)

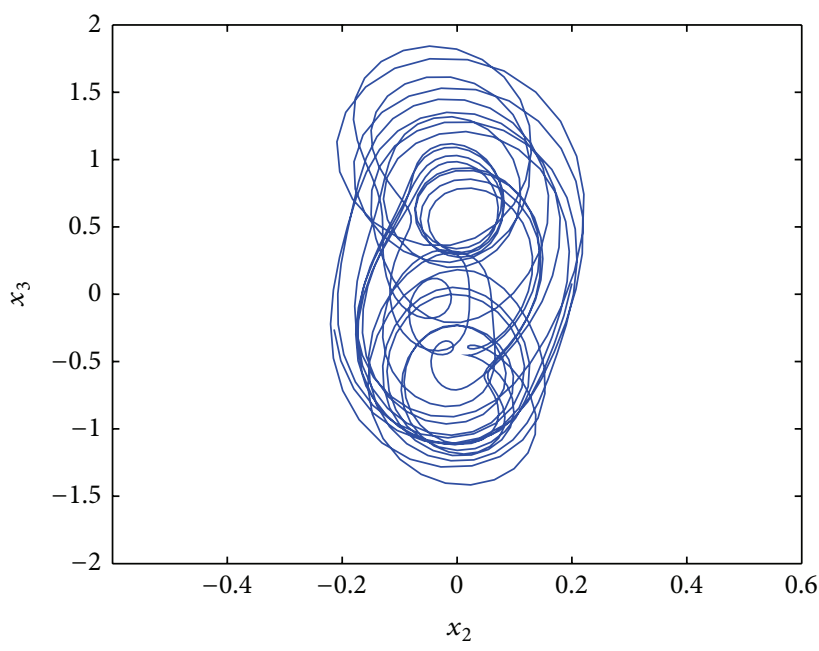

(d)

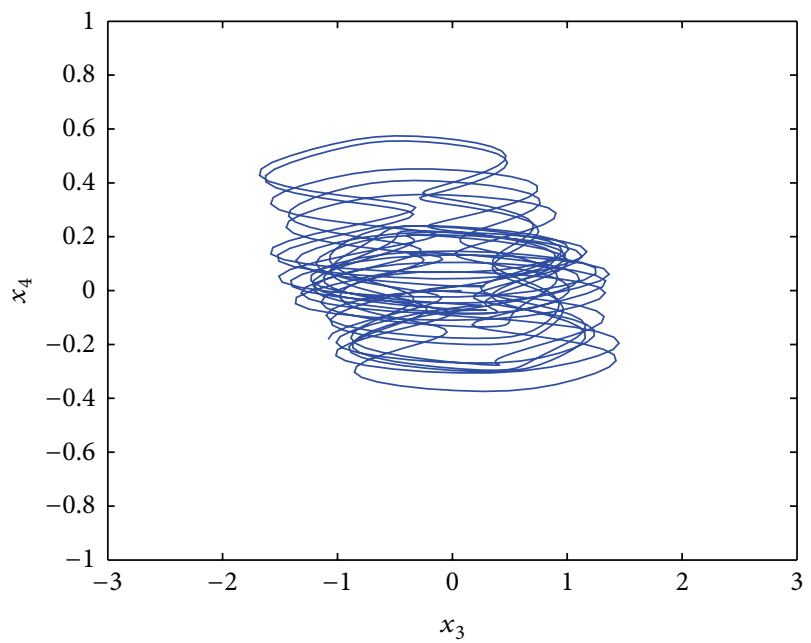

(f)

FIGURE 8: Fractional-order phase portraits of hyperchaotic attractors on $x_{1}-x_{2}, x_{1}-x_{3}, x_{1}-x_{4}, x_{2}-x_{3}, x_{2}-x_{4}$, and $x_{3}-x_{4}$ plane projection. 


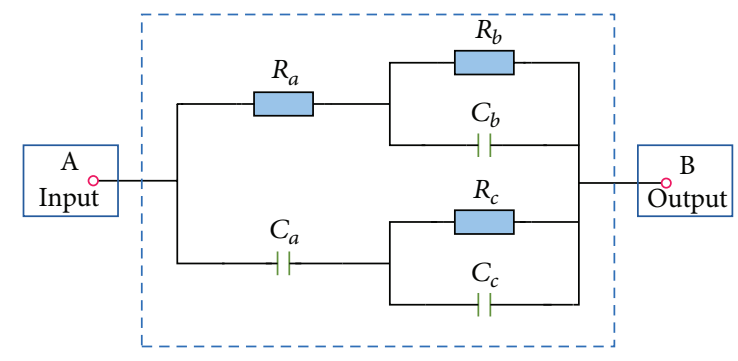

FIGURE 9: Network-type fractance module in three pairs.

Therefore, the numerical solution of a general fractionalorder system can be obtained by using the aforementioned algorithm.

When assuming the fractional-order $\alpha=0.9$, the fractional-order chaotic attractors have been obtained for determining that the system (2) is fractal. Figure 8 illustrates the phase diagram of fractional chaotic attractors in different plane.

3.2. Nonlinear Circuit Verification for Fractional-Order Hyperchaotic System. As is well known, the standard definition of fractional differintegral does not allow direct implementation of the fractional operators in time-domain simulations. In order to design the circuit of fractional-order form of dynamical system, one can obtain a linear approximation of a fractional-order integrator with any desired accuracy over any frequency band based on Bode diagrams. Generally, a fractional dynamical power function dependence on frequency can be obtained by approximations as $H(s)=1 / S^{\alpha}$ with $\alpha=0.1 \sim 0.9$ in step size 0.1 . Hence a typical multiple fractional power pole model can be described as

$$
H(s)=\frac{1}{\prod_{i=1}^{P}\left(1+\left(s / p_{i} T_{i}\right)\right)^{m_{i}}}, \quad 0<m_{i}<1 ; i=1,2, \ldots, p,
$$

where $1 / p_{i} T_{i}$ is the relaxation time constants. The approximated transfer function can be written as follows:

$$
H(s)=\frac{1}{\prod_{i=1}^{P}\left(1+\left(s / p_{i} T_{i}\right)\right)^{m_{i}}} \approx \frac{\prod_{i=0}^{N-1}\left(1+\left(s / z_{i}\right)\right)}{\prod_{i=0}^{N}\left(1+\left(s / p_{i}\right)\right)},
$$

where $N$ is a finite number and $p_{i}$ and $z_{i}$ are pole and zero in the geometrical progression, respectively.

From the above derivations, according to Table 1 provided in [34], the approximation transfer function of $1 / s^{0.9}$ with an error of $2 \mathrm{~dB}$ is given as follows:

$$
\frac{1}{S^{0.9}} \approx \frac{2.2675(s+1.292)(s+215.4)}{(s+0.01292)(s+2.154)(s+359.4)} .
$$

There are two types of electrical circuit modules which are related to the fractional calculus. The first model is the tree-type fractance element where an infinite self-similar circuit consisting of resistors of resistance $R$ and capacitors of capacitance $C$ is depicted. The second model is the chain-type fractance which consists of resistor-capacitor pairs connected in a chain. To generate fractional-order attractors from system (12), we introduce a new network-type fractance module which is different from traditional tree and chaintype fractional fractance. The new fractance consists of $N$ resistor-capacitor pairs connected in a network. As for tree and chain fractance, the network fractance can also be used for analogue fractional differentiation and integration and as a circuit module of a new type. In practical applications, the circuit network (Figure 9) has been chosen as three $R C$ pairs by using resistors $R_{a}, R_{b}$, and $R_{c}$ and linear capacitors $C_{a}, C_{b}$, and $C_{c}$.

The corresponding transfer function $H(s)$ of this circuit module can be written into

$H(s)$

$$
\begin{aligned}
= & {\left[R_{a}+\left(R_{b} / / \frac{1}{s C_{b}}\right)\right] / /\left[\frac{1}{s C_{a}}+\left(R_{c} / / \frac{1}{s C_{c}}\right)\right] } \\
= & \left(\left(\frac{C_{0}}{C_{a}}+\frac{C_{0}}{C_{c}}\right)\left(s+\frac{R_{a}+R_{b}}{R_{a} C_{b} R_{b}}\right)\left(s+\frac{1}{C_{a} R_{c}+C_{c} R_{c}}\right)\right) \\
& \times\left(C_{0} s^{3}+\left[\frac{C_{0}\left(R_{a}+R_{b}\right)}{R_{a} C_{b} R_{b}}+\frac{C_{0}}{C_{c} R_{c}}+\frac{C_{0}\left(C_{a}+C_{c}\right)}{C_{a} R_{a} C_{c}}\right] s^{2}\right. \\
& \left.+\left[\frac{C_{0}\left(R_{a}+R_{b}\right)}{R_{a} C_{b} R_{b} C_{c} R_{c}}+\frac{C_{0}}{C_{a} R_{a} C_{c} R_{c}}+\frac{C_{0}\left(C_{a}+C_{c}\right)}{C_{a} R_{a} C_{b} R_{b} C_{c}}\right] s\right)^{-1} \\
& +\left(\left(\frac{C_{0}}{C_{a}}+\frac{C_{0}}{C_{c}}\right)\left(s+\frac{R_{a}+R_{b}}{R_{a} C_{b} R_{b}}\right)\left(s+\frac{1}{C_{a} R_{c}+C_{c} R_{c}}\right)\right)^{-1} \\
& \times\left(\frac{C_{0}}{C_{a} R_{a} C_{b} R_{b} C_{c} R_{c}}\right)^{-1}
\end{aligned}
$$

where $C_{0}$ is a fixed-value parameter. In practice, one can obtain the transfer function as $F(s)=H(s) C_{0}=1 / s^{0.9}$ by adjusting a value of $C_{0}$ as $C_{0}=1 \mu \mathrm{F}$. In addition, comparing $F(s)$ with $E_{q}$. (26), some adjustable resistances can be tuned as $R_{a}=1.55 \mathrm{M} \Omega, R_{b}=61.54 \mathrm{M} \Omega, R_{c}=2.526 \mathrm{k} \Omega, C_{a}=$ $0.734 \mu \mathrm{F}, C_{b}=0.522 \mu \mathrm{F}$, and $C_{c}=1.103 \mu \mathrm{F}$.

Furthermore, to verify the observed strange attractors from the fractional-order form of hyperchaotic system, an experimental nonlinear fourth-channel fractional circuit has been designed which included two main parts; that is, the first part shows the network-type fractance module in Figure 9 and the second part shows the integer-order circuit in Figure 6. The full schematic diagram with detailed specifications is depicted in Figure 10.

In addition, the corresponding exact state $R$ - $C$ equations can be derived as follows:

$$
\begin{aligned}
\frac{d^{0.9} u_{C_{1}}}{d t^{0.9}}= & \frac{R_{2}}{R_{3} R_{7} C_{1}} u_{C_{2}}+\frac{R_{2}}{R_{3} C_{1}}\left(\frac{1}{R_{6}} u_{C_{1}}-\frac{R_{5}}{100 R_{1} R_{4}} u_{C_{1}}^{3}\right), \\
\frac{d^{0.9} u_{C_{2}}}{d t^{0.9}}= & \frac{R_{9}}{R_{8} R_{10} C_{2}} u_{C_{1}}-\frac{R_{9} R_{12}}{R_{10} R_{11} R_{13} C_{2}} u_{C_{2}}+\frac{R_{9}}{R_{10} R_{14} C_{2}} u_{C_{3}} \\
& +\frac{R_{9}}{R_{10} R_{18} C_{2}} u_{C_{4}},
\end{aligned}
$$




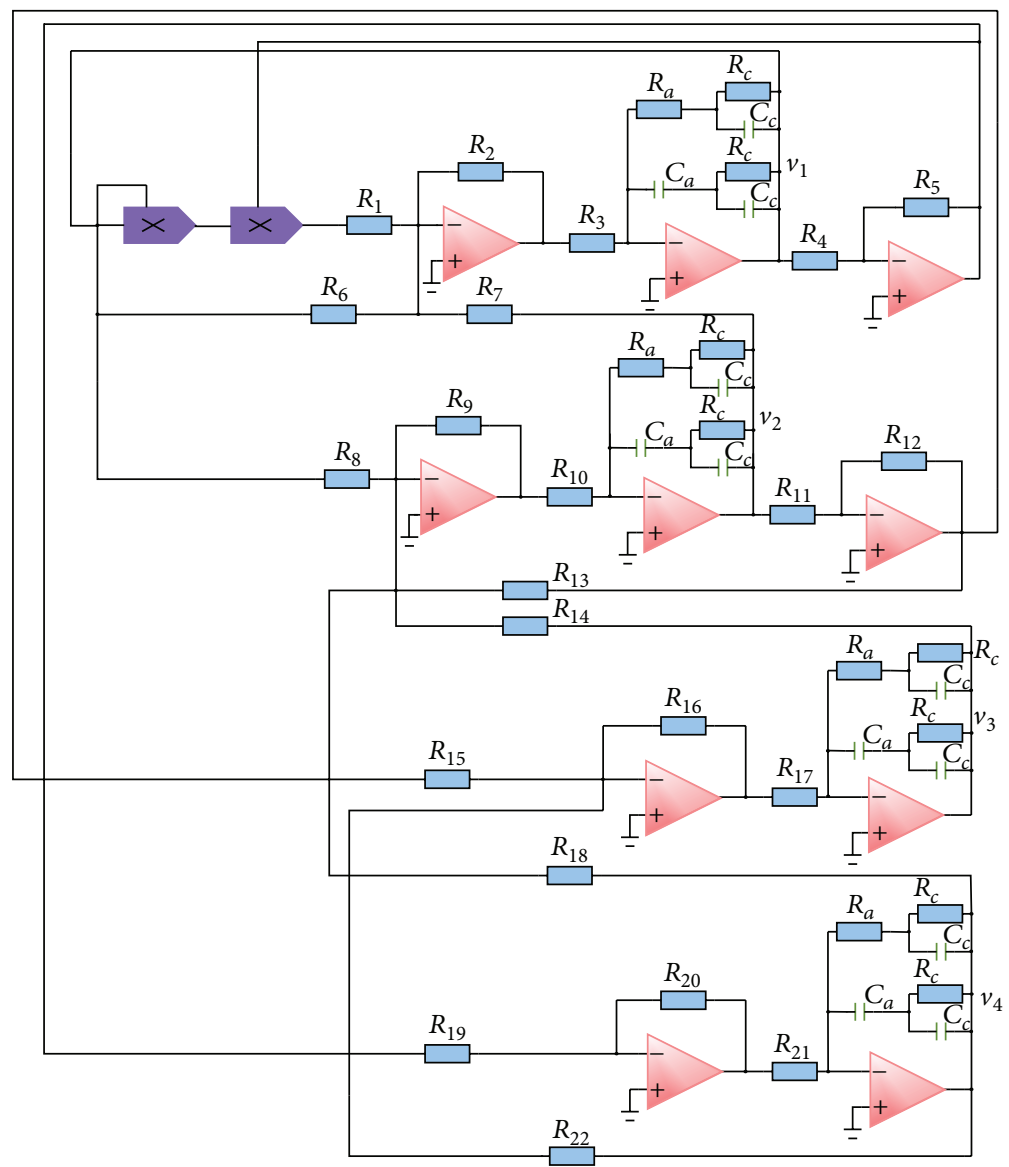

FIGURE 10: Circuit diagram for implementing fractional-order hyperchaotic attractors.

$$
\begin{aligned}
& \frac{d^{0.9} u_{C_{3}}}{d t^{0.9}}=-\frac{R_{12} R_{16}}{R_{11} R_{15} R_{17} C_{3}} u_{C_{2}}-\frac{R_{16}}{R_{17} R_{22} C_{3}} u_{C_{4}}, \\
& \frac{d^{0.9} u_{C_{4}}}{d t^{0.9}}=-\frac{R_{5} R_{20}}{R_{4} R_{19} R_{21} C_{4}} u_{C_{1}},
\end{aligned}
$$

where $C_{1} \sim C_{4}$ should be replaced by the network-type fractance.

In experiment, assume that the supply voltages are chosen as $V= \pm 15 \mathrm{~V}$. Operational amplifiers and analog multipliers are chosen as in integer-order circuit. Digital oscilloscope TEK TPS2014 is used to detect the phase plane and waveforms. Figures 11(a)-11(f) show the oscilloscope-observed results for fractional-order of chaotic attractors. Moreover, comparing Figure 8 with Figure 11, very good qualitative agreement between the integer attractors and fractionalorder attractors can be observed.

\section{Conclusions}

In this paper, a new four-dimensional nonlinear smooth autonomous hyperchaotic system derived from the famous
Chua circuit has been investigated in detail. Some dynamical behaviors of this system are analysis by means of equilibrium point, bifurcations, Poincaré mapping, parameter phase portraits, and calculated Lyapunov exponents. Meanwhile, a simple fourth-channel circuit diagram has been designed for verifying these new chaotic attractors. In particular, based on the fractional-order theorem, we have presented a novel network element fractance which can be used by designing a novel nonlinear fractional-order circuit. Our physical experiment results demonstrate the effectiveness of this fractional-order circuit design.

It is assumed that there still are some abundant and complex dynamical properties unknown in this modified fourdimensional Chua's system. This will greatly enhance our understanding of chaotic systems in general and implement fractional-order chaotic attractors using hardware devices in the near future.

\section{Conflict of Interests}

The authors declare that there is no conflict of interests regarding the publication of this paper. 


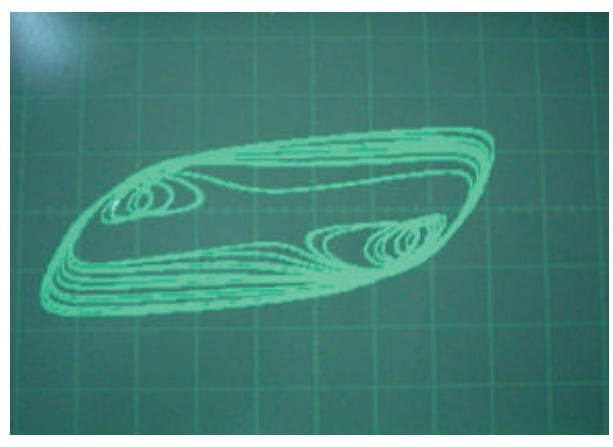

(a) $x_{1}-x_{2}$

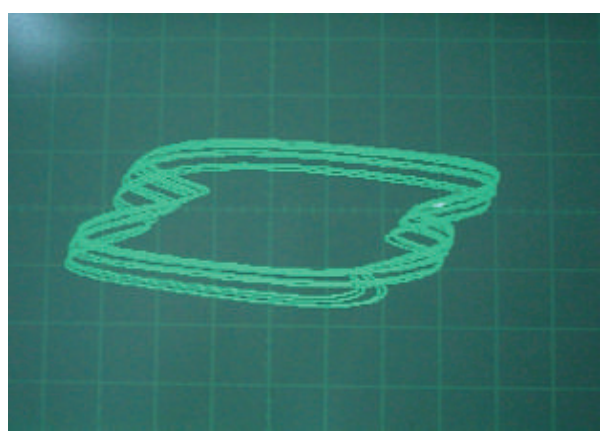

(c) $x_{1}-x_{4}$

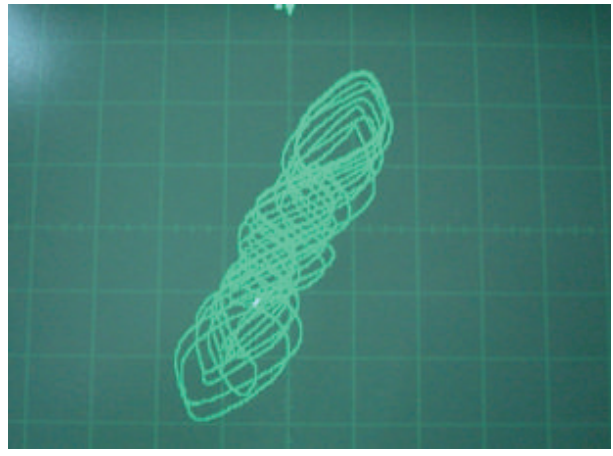

(e) $x_{2}-x_{4}$

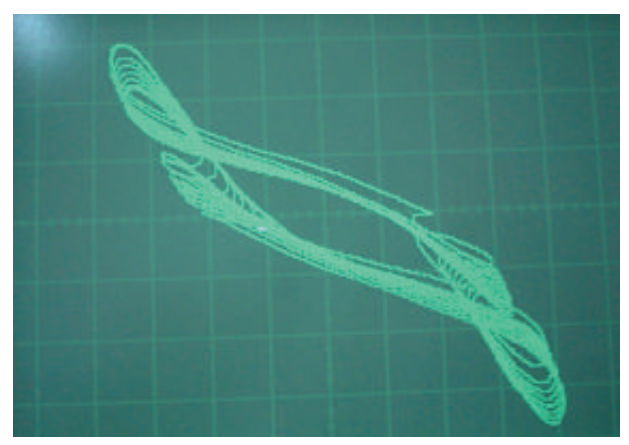

(b) $x_{1}-x_{3}$

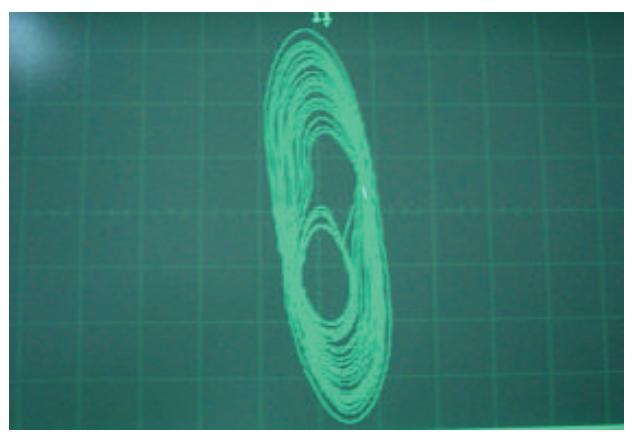

(d) $x_{2}-x_{3}$

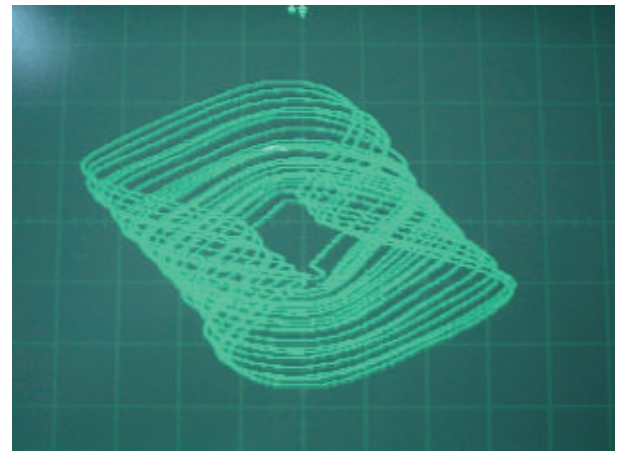

(f) $x_{3}-x_{4}$

FIGURE 11: Experimental observations of the fractional-order hyperchaotic attractors with $\alpha=0.9$.

\section{Acknowledgment}

This work was supported by the National Natural Science Foundation of China (Grant no. 51307130).

\section{References}

[1] E. N. Lorenz, "Deterministic nonperiodic flow," Journal of the Atmospheric Sciences, vol. 20, pp. 130-141, 1963.

[2] L. O. Chua, M. Komuro, and T. Matsumoto, "The double scroll family," IEEE Transactions on Circuits and Systems, vol. 33, no. 11, pp. 1072-1118, 1986.

[3] G. Chen and T. Ueta, "Yet another chaotic attractor," International Journal of Bifurcation and Chaos in Applied Sciences and Engineering, vol. 9, no. 7, pp. 1465-1466, 1999.

[4] J. Lü and G. Chen, "A new chaotic attractor coined," International Journal of Bifurcation and Chaos, vol. 12, no. 3, pp. 659661, 2002.
[5] C. Liu, T. Liu, L. Liu, and K. Liu, "A new chaotic attractor," Chaos, Solitons and Fractals, vol. 22, no. 5, pp. 1031-1038, 2004.

[6] G. Qi, G. Chen, S. Du, Z. Chen, and Z. Yuan, "Analysis of a new chaotic system," Physica A: Statistical Mechanics and Its Applications, vol. 352, no. 2-4, pp. 295-308, 2005.

[7] L. Liu, Y. Su, C. Liu, and T. Liu, "A modified Lorenz system," International Journal of Nonlinear Sciences and Numerical Simulation, vol. 7, no. 2, pp. 187-190, 2006.

[8] L. Liu, Y. C. Su, and C. X. Liu, "A new chaotic system and its circuit emulation," Acta Physica Sinica, vol. 55, no. 8, pp. 39333905, 2006.

[9] Z. Chen, Y. Yang, G. Qi, and Z. Yuan, "A novel hyperchaos system only with one equilibrium," Physics Letters A, vol. 360, no. 6, pp. 696-701, 2007.

[10] L. Liu, Y. Su, and C. Liu, "Experimental confirmation of a new reversed butterfly-shaped attractor," Chinese Physics, vol. 16, no. 7, pp. 1897-1904, 2007. 
[11] J. Cheng, H. Zhu, S. Zhong, Y. Zeng, and X. Dong, "Finite-time $\mathrm{H} \infty$ control for a class of Markovian jump systems with modedependent time-varying delays via new Lyapunov functionals," ISA Transactions, vol. 52, no. 6, pp. 768-774, 2013.

[12] J. Cheng, H. Zhu, Y. Ding, S. Zhong, and Q. Zhong, "Stochastic finite-time boundedness for Markovian jumping neural networks with time-varying delays," Applied Mathematics and Computation, vol. 242, no. 1, pp. 281-295, 2014.

[13] A. Čenys, A. Tamaševičius, A. Baziliauskas, R. Krivickas, and E. Lindberg, "Hyperchaos in coupled Colpitts oscillators," Chaos, Solitons and Fractals, vol. 17, no. 2-3, pp. 349-353, 2003.

[14] Y. Li, W. K. S. Tang, and G. Chen, "Hyperchaos evolved from the generalized Lorenz equation," International Journal of Circuit Theory and Applications, vol. 33, no. 4, pp. 235-251, 2005.

[15] G. Qi and G. Chen, "Analysis and circuit implementation of a new 4D chaotic system," Physics Letters A, vol. 352, no. 4-5, pp. 386-397, 2006.

[16] F. Wang and C. Liu, "Hyperchaos evolved from the Liu chaotic system," Chinese Physics, vol. 15, no. 5, pp. 963-968, 2006.

[17] J. Lü and G. Chen, "Generating multiscroll chaotic attractors: theories, methods and applications," International Journal of Bifurcation and Chaos, vol. 16, no. 4, pp. 775-858, 2006.

[18] L. Liu, C. Liu, and Y. Zhang, "Experimental confirmation of a modified Lorenz system," Chinese Physics Letters, vol. 24, no. 10, pp. 2756-2758, 2007.

[19] L. Chong-Xin and L. Ling, "A novel four-dimensional autonomous hyperchaotic system," Chinese Physics B, vol. 18, no. 6, pp. 2188-2193, 2009.

[20] L. Liu, C. Liu, and Y. Zhang, "Theoretical analysis and circuit implementation of a novel complicated hyperchaotic system," Nonlinear Dynamics, vol. 66, no. 4, pp. 707-715, 2011.

[21] T. T. Hartley, C. F. Lorenzo, and H. K. Qammer, "Chaos in a fractional order Chua's system," IEEE Transactions on Circuits and Systems I, vol. 42, no. 8, pp. 485-490, 1995.

[22] I. Grigorenko and E. Grigorenko, "Chaotic dnamics of the fractional Lorenz system," Physical Review Letters, vol. 91, pp. 101-104, 2003.

[23] Z. Ge and C. Ou, "Chaos in a fractional order modified Duffing system," Chaos, Solitons and Fractals, vol. 34, no. 2, pp. 262-291, 2007.

[24] C. Li and G. Chen, "Chaos and hyperchaos in the fractionalorder Rössler equations," Physica A: Statistical Mechanics and its Applications, vol. 341, no. 1-4, pp. 55-61, 2004.

[25] W. Deng and C. Li, "The evolution of chaotic dynamics for fractional unified system," Physics Letters A: General, Atomic and Solid State Physics, vol. 372, no. 4, pp. 401-407, 2008.

[26] M. Nakagawa and K. Sorimachi, "Basic characteristics of a fractance device," IEICE Transactions on Fundamentals of Electronics, vol. 75, no. 12, pp. 1814-1819, 1992.

[27] K. B. Oldham and C. G. Zoski, "Analogue instrumentation for processing polarographic data," Journal of Electroanalytical Chemistry, vol. 157, no. 1, pp. 27-51, 1983.

[28] J. J. Lu and C. X. Liu, "Realization of fractional-order Liu chaotic system by circuit," Chinese Physics B, vol. 16, no. 6, pp. 15861590, 2007.

[29] C. Liu and L. Liu, "Circuit implementation of a new hyperchaos in fractional-order system," Chinese Physics B, vol. 17, no. 8, pp. 2829-2837, 2008.

[30] L. Liu, C. Liu, and Y. Zhang, "Experimental verification of a four-dimensional chua's system and its fractional order chaotic attractors," International Journal of Bifurcation and Chaos, vol. 19, no. 8, pp. 2473-2486, 2009.

[31] S. Dadras, H. R. Momeni, G. Qi, and Z. Wang, "Fourwing hyperchaotic attractor generated from a new $4 \mathrm{D}$ system with one equilibrium and its fractional-order form," Nonlinear Dynamics, vol. 67, no. 2, pp. 1161-1173, 2012.

[32] J. W. Cao, "Improved delay-dependent stability conditions for MIMO networked control systems with nonlinear perturbations," The Scientific World Journal, vol. 2014, Article ID 196927, 4 pages, 2014.

[33] J. Cao, "Improved delay-dependent exponential stability criteria for time-delay system," Journal of the Franklin Institute, vol. 350, no. 4, pp. 790-801, 2013.

[34] W. M. Ahmad and A. M. Harb, "On nonlinear control design for autonomous chaotic systems of integer and fractional orders," Chaos, Solitons and Fractals, vol. 18, no. 4, pp. 693-701, 2003. 


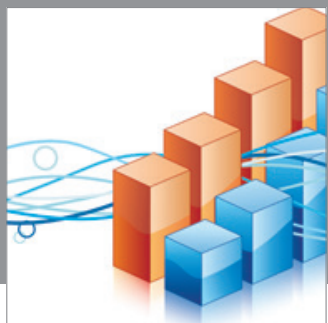

Advances in

Operations Research

mansans

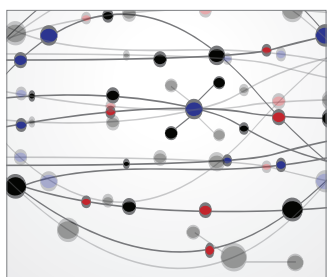

The Scientific World Journal
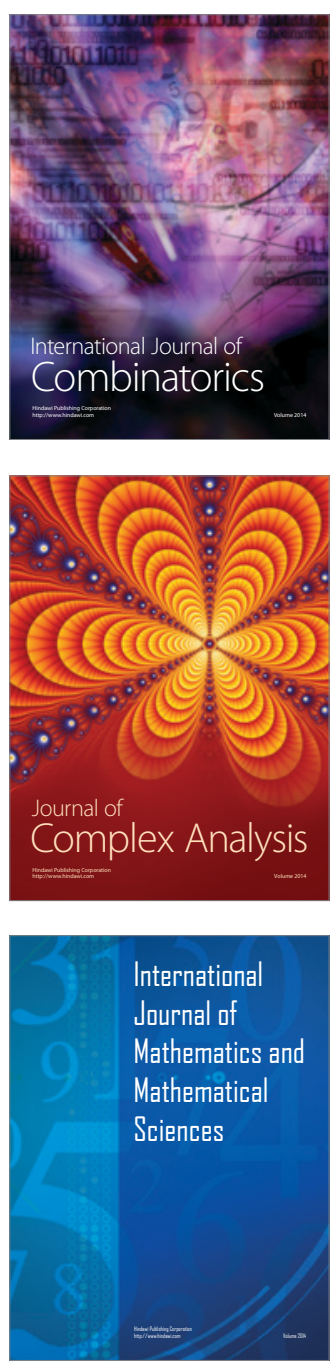
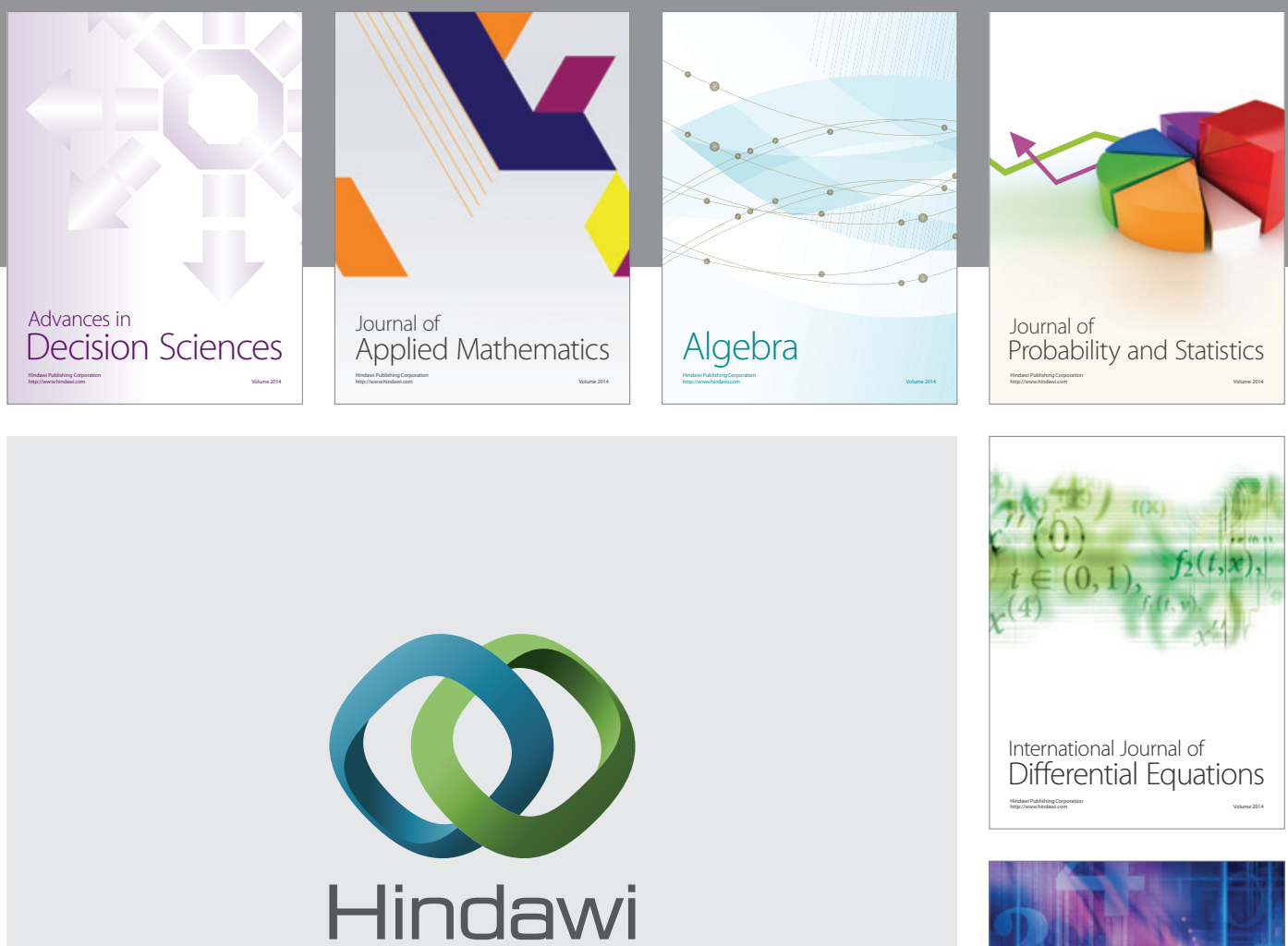

Submit your manuscripts at http://www.hindawi.com
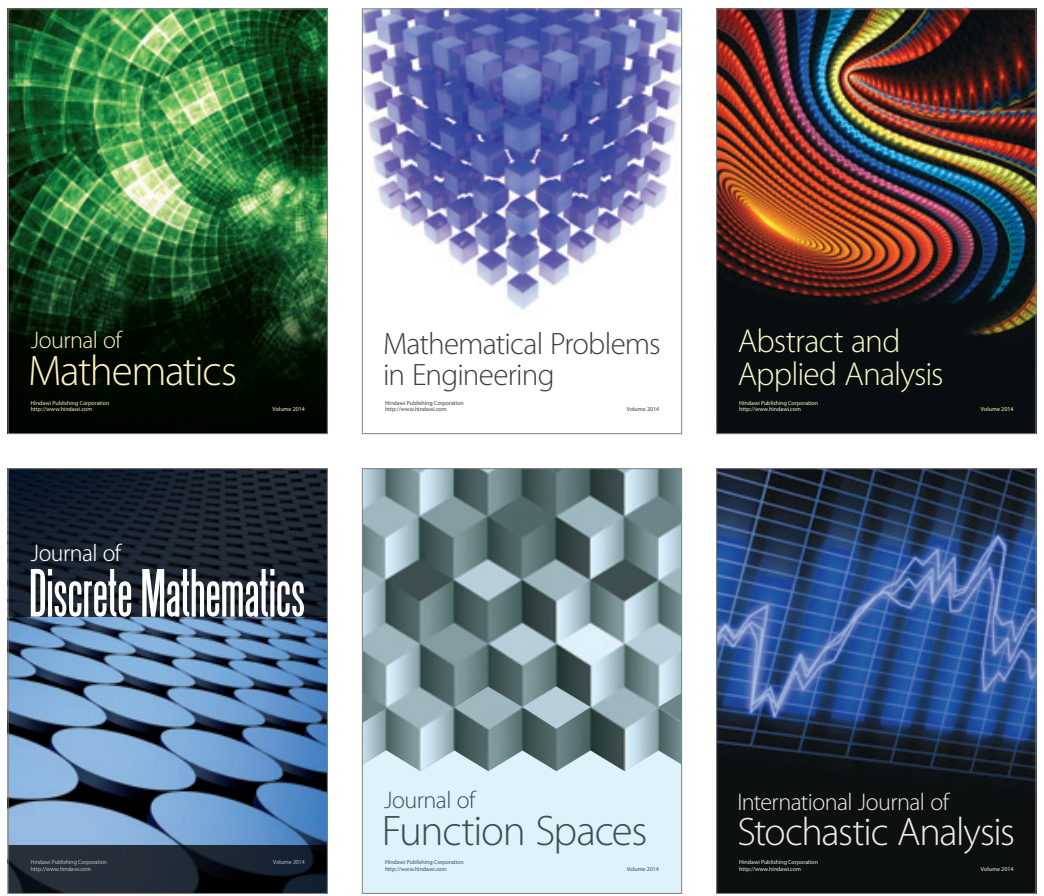

Journal of

Function Spaces

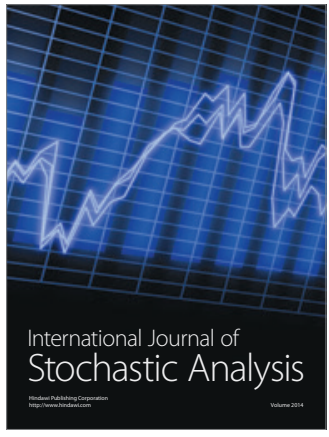

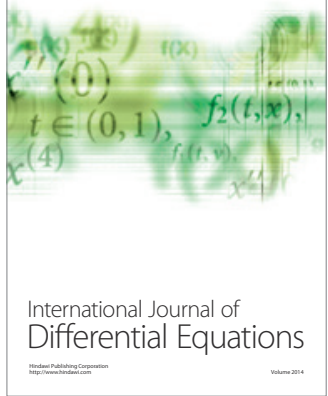
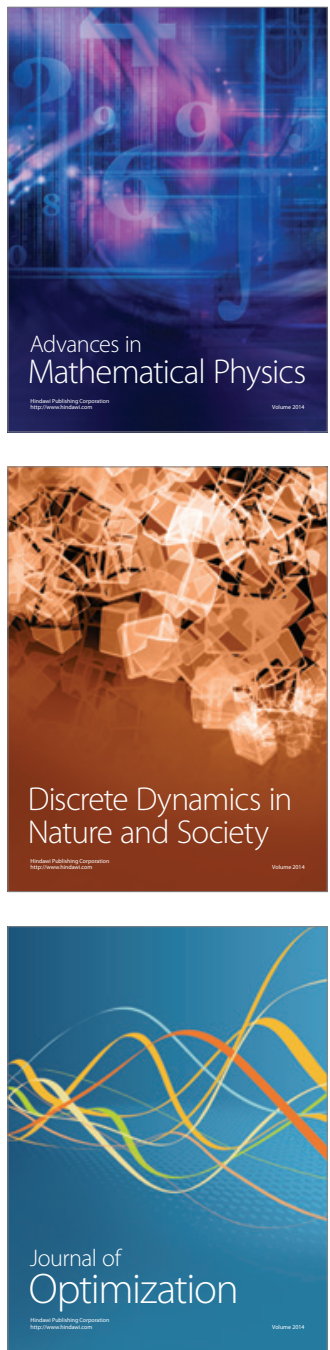